NASA/TM-2013-217971

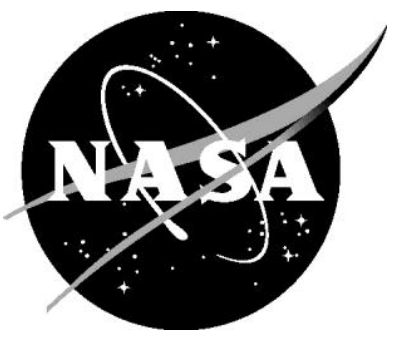

High-Order Entropy Stable Finite Difference Schemes for Nonlinear Conservation Laws: Finite Domains

Travis C. Fisher and Mark H. Carpenter

Langley Research Center, Hampton, Virginia 


\section{NASA STI Program ... in Profile}

Since its founding, NASA has been dedicated to the advancement of aeronautics and space science. The NASA scientific and technical information (STI) program plays a key part in helping NASA maintain this important role.

The NASA STI program operates under the auspices of the Agency Chief Information Officer. It collects, organizes, provides for archiving, and disseminates NASA's STI. The NASA STI program provides access to the NASA Aeronautics and Space Database and its public interface, the NASA Technical Report Server, thus providing one of the largest collections of aeronautical and space science STI in the world. Results are published in both non-NASA channels and by NASA in the NASA STI Report Series, which includes the following report types:

- TECHNICAL PUBLICATION. Reports of completed research or a major significant phase of research that present the results of NASA Programs and include extensive data or theoretical analysis. Includes compilations of significant scientific and technical data and information deemed to be of continuing reference value. NASA counterpart of peerreviewed formal professional papers, but having less stringent limitations on manuscript length and extent of graphic presentations.

- TECHNICAL MEMORANDUM. Scientific and technical findings that are preliminary or of specialized interest, e.g., quick release reports, working papers, and bibliographies that contain minimal annotation. Does not contain extensive analysis.

- CONTRACTOR REPORT. Scientific and technical findings by NASA-sponsored contractors and grantees.
- CONFERENCE PUBLICATION. Collected papers from scientific and technical conferences, symposia, seminars, or other meetings sponsored or cosponsored by NASA.

- SPECIAL PUBLICATION. Scientific, technical, or historical information from NASA programs, projects, and missions, often concerned with subjects having substantial public interest.

- TECHNICAL TRANSLATION. English-language translations of foreign scientific and technical material pertinent to NASA's mission.

Specialized services also include organizing and publishing research results, distributing specialized research announcements and feeds, providing information desk and personal search support, and enabling data exchange services.

For more information about the NASA STI program, see the following:

- Access the NASA STI program home page at http://www.sti.nasa.gov

- E-mail your question to help@sti.nasa.gov

- Fax your question to the NASA STI Information Desk at 443-757-5803

- Phone the NASA STI Information Desk at 443-757-5802

- Write to:

STI Information Desk NASA Center for AeroSpace Information 7115 Standard Drive Hanover, MD 21076-1320 
NASA/TM-2013-217971

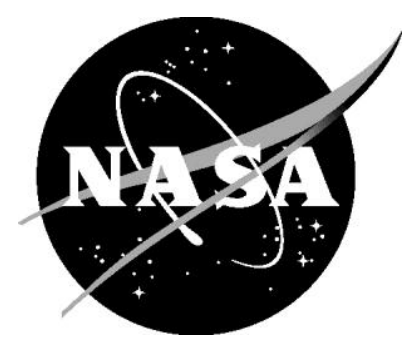

\title{
High-Order Entropy Stable Finite Difference Schemes for Nonlinear Conservation Laws: Finite Domains
}

\author{
Travis C. Fisher and Mark H. Carpenter
}

Langley Research Center, Hampton, Virginia

National Aeronautics and

Space Administration

Langley Research Center

Hampton, Virginia 23681-2199

February 2013 


\section{Acknowledgments}

This work summarizes portions of first author's Ph. D. dissertation, performed as a cooperative student while in residence at NASA Langley Research Center. Special thanks are extended to Dr. Mujeeb Malik for funding the cooperative agreement as part of the "Revolutionary Computational Aerosciences" project. Special thanks are also extended to Dr. Nail Yamaleev for proof-reading the document and for correcting formula (3.42).

The use of trademarks or names of manufacturers in this report is for accurate reporting and does not constitute an official endorsement, either expressed or implied, of such products or manufacturers by the National Aeronautics and Space Administration

Available from:

NASA Center for AeroSpace Information

7115 Standard Drive

Hanover, MD 21076-1320

443-757-5802 


\begin{abstract}
Developing stable and robust high-order finite difference schemes requires mathematical formalism and appropriate methods of analysis. In this work, nonlinear entropy stability is used to derive provably stable high-order finite difference methods with formal boundary closures for conservation laws. Particular emphasis is placed on the entropy stability of the compressible Navier-Stokes equations. A newly derived entropy stable weighted essentially non-oscillatory finite difference method is used to simulate problems with shocks and a conservative, entropy stable, narrowstencil finite difference approach is used to approximate viscous terms.
\end{abstract}

\title{
Contents
}

1 Introduction 2

2 Methodology 3

2.1 Nonlinear Conservation Laws and the Entropy Condition . . . . . . 3

2.2 Entropy Analysis . . . . . . . . . . . . . . . 5

2.3 Spatial Discretization . . . . . . . . . . . . . 7

2.3.1 Complementary Grids . . . . . . . . . . . . . . 7

2.3.2 First Derivative Approximation . . . . . . . . . . . . 9

2.3.3 Variable Coefficient Second Derivative Approximation . . . . 9

2.3.4 Telescopic Flux Form . . . . . . . . . . . . . . . . . . 11

2.3.5 Semi-Discretization . . . . . . . . . . . . . . 12

2.4 Satisfying the Weak Form . . . . . . . . . . . . . . . 12

2.5 Temporal Integration . . . . . . . . . . . . . . . . . . 13

3 Entropy Stable Finite Differences $\quad 13$

3.1 Semi-Discrete Entropy Analysis . . . . . . . . . . . . . . . . . . 13

3.2 Inviscid Flux Conditions . . . . . . . . . . . . . . . . . . . . . 14

3.2.1 Entropy Consistent Fluxes . . . . . . . . . . . . . . . . . . 14

3.2 .2 Entropy Stability . . . . . . . . . . . . . . . 20

3.3 Entropy Stable WENO Finite Differences . . . . . . . . . . . . . . . 21

3.4 Entropy Stable Viscous Terms . . . . . . . . . . . . . . . . . . 23

3.5 Entropy Stable Semi-Discretization . . . . . . . . . . . . . . . 24

4 Applications $\quad \mathbf{2 4}$

4.1 Burgers Equation . . . . . . . . . . . . . . . . 25

4.1.1 Entropy Stable Discretization of Burgers Equation . . . . . . 26

4.1.2 Advantages of Entropy Stability . . . . . . . . . . . 27

4.2 Euler and Navier-Stokes Equations . . . . . . . . . . . . . . . 28

4.2 .1 Entropy Analysis . . . . . . . . . . . . . . . . . . . 30

4.2 .2 Discretization Notes . . . . . . . . . . . . . . . . . . . 31

4.2.3 Entropy Stable Spatial Discretization . . . . . . . . . . . 32

4.2.4 Energy Stable Boundary Conditions . . . . . . . . . . . . 35 
5 Accuracy Validation and Robustness $\quad \mathbf{3 5}$

5.1 Isentropic Vortex . . . . . . . . . . . . . . . . . 35

5.1.1 Optimal Accuracy: A Periodic Cartesian Grid Test Case . . . 36

5.1 .2 Finite Domain Cartesian Grid Test . . . . . . . . . . . . 36

5.2 Viscous Shock . . . . . . . . . . . . . . . 37

5.3 Shock Tube Problems . . . . . . . . . . . . . . . . . . . 38

5.3 .1 Sod Shock Tube . . . . . . . . . . . . . . 39

5.3 .2 Lax Shock Tube . . . . . . . . . . . . . . . . . . . . 39

6 Conclusions $\quad 40$

A Summation-by-parts operators-(2-4-2) 44

A.1 First Derivative . . . . . . . . . . . . . . . . . . 44

A.1.1 Flux Form . . . . . . . . . . . . . . . 45

A.2 Variable Coefficient Second Derivative . . . . . . . . . . . . 45

A.2.1 Flux Form . . . . . . . . . . . . . . . . . 48

B Navier-Stokes Equations-Supplemental Details $\mathbf{5 2}$

B.1 Derivation of Entropy Variables . . . . . . . . . . . . . . . . 52

B.2 Viscous Stability . . . . . . . . . . . . . . 53

\section{Introduction}

The state of numerical solutions to nonlinear conservation laws is far from complete. While it is commonplace to use high-order numerical methods to calculate efficient and accurate solutions for smooth problems, solutions of problems with shocks are considerably more difficult to simulate. Solution methods for these problems are typically high-order adaptive $[1,2]$ or hybrid [3] schemes or highly dissipative loworder methods. Many methods have been devised that attempt to balance accuracy, added dissipation, and efficiency. Most of these methods are designed using linear analysis of linearized equations that do not admit the formation of shocks and thus do not correctly account for the character of the underlying nonlinear problem. Additionally, stability proofs that rely on linear analysis are dependent on the resolution and do not guarantee stability for under-resolved regions. To overcome these limitations, we seek numerical methods that are based on nonlinear analysis.

Any numerical method applied to problems that admit shocks should provably recover the weak solution of the conservation law upon convergence [4]. It should be further proven that the weak solution recovered is the physically realizable entropy solution $[4,5]$. The second condition is an uncommon property in high-order methods. Recent advancements in this area for the compressible Euler equations facilitate incorporation of these properties into high-order formulations. Tadmor constructed entropy consistent second-order finite volume schemes that conserve discrete entropy $[5,6]$, while LeFloch and Rode [7] extended these schemes to highorder periodic domains. These schemes have been made computationally tractable 
for the Navier-Stokes equations through the work of Ismail and Roe [8]. A methodology for constructing entropy stable schemes satisfying a cell entropy inequality and capable of simulating flows with shocks in periodic domains has been developed by Fjordholm et al. [9] Herein, an alternative approach is developed based on a finitedomain entropy stability proof, which yields entropy stable methods with formal boundary closures.

In this work, we have constructed high-order entropy stable finite difference schemes for finite domains by first developing a formal set of conditions based on a generalized summation-by-parts property. The entropy consistent scheme for conservation laws developed by Tadmor [6] is extended to high-order with formal boundary closures. Based on this new entropy consistent scheme, we develop an entropy stable correction for dissipative numerical methods such as weighted essentially nonoscillatory (WENO) for simulating problems with shocks. Additionally, we have derived a narrow-stencil, high-order viscous operator for approximating the viscous terms in a provably entropy stable manner.

Using the methodology developed herein, we demonstrate the robustness and accuracy of the resulting entropy stable WENO operators using Burgers equation and the Euler equations. We also show how schemes developed using linear stability can fail in the presence of a shock by comparing the newly developed entropy stable schemes with the energy stable WENO scheme of Fisher et al. [10].

Results of the present work warrant investigation into the extension of the current entropy-stable numerical methods into generalized curvilinear coordinates.

The organization of this document is as follows. The theory of entropy analysis for finite difference methods is detailed in Section 2. Conditions and corresponding methods for satisfying entropy stability on finite domains using arbitrarily highorder accurate finite difference methods are developed in Section 3. The application of these methods to Burgers equation and the compressible Euler and Navier-Stokes equations is illustrated in Section 4. Finally, the accuracy and robustness of the resulting high-order schemes are demonstrated in Section 5, and conclusions are discussed in Section 6.

\section{Methodology}

In this section, we introduce the theory of entropy stability and define the necessary finite difference nomenclature for conservation laws.

\subsection{Nonlinear Conservation Laws and the Entropy Condition}

The most general form of the one-dimensional inviscid conservation law on a bounded domain is the integral form,

$$
\frac{d}{d t} \int_{x_{L}}^{x_{R}} q \mathrm{~d} x+\left.f(q)\right|_{x_{L}} ^{x_{R}}=0, \quad x \in\left[x_{L}, x_{R}\right], \quad t \in[0, \infty),
$$


where $q$ denotes a scalar or vector of conserved variables, $f$ is the nonlinear flux function, and the domain bounds have been assumed fixed. As noted by Lax [11], solutions satisfying the integral form in 2.1 are generalized or weak solutions of the conservation law and do not need to be smooth or even continuous. For smooth problems, the strong differential form of the conservation law can be written as

$$
q_{t}+f(q)_{x}=0, \quad x \in\left[x_{L}, x_{R}\right], \quad t \in[0, \infty) .
$$

The solution to 2.2, referred to as a strong solution, is smooth and unique but may not exist for all time if the physical solution becomes discontinuous. The strong solution also satisfies 2.1.

Piecewise continuous solutions to the integral form of the conservation law must satisfy the strong form on either side of a discontinuity. Additionally, the Rankine Hugoniot relation holds across discontinuities [11],

$$
[f(q)]_{\Gamma_{d}^{-}}^{\Gamma_{d}^{+}}-\frac{d \Gamma_{d}}{d t}[q]_{\Gamma_{d}^{-}}^{\Gamma_{d}^{+}}=0
$$

where $\Gamma_{d}$ denotes the discontinuity location and $\frac{d \Gamma_{d}}{d t}$ denotes the propagation speed of the discontinuity. These characteristics are derived directly from the integral form.

Note that weak solutions in general may not be unique $[11,12]$, and that only the physically realizable entropy solution is of interest. This solution is described through a limiting process of a regularized conservation law that admits a strong solution for all time, $q^{\varepsilon}(x, t)$, satisfying [12]

$$
q_{t}^{\varepsilon}+f\left(q^{\varepsilon}\right)_{x}=\varepsilon\left(f^{(v)}\left(q^{\varepsilon}, q_{x}^{\varepsilon}\right)\right)_{x},
$$

where $\varepsilon>0$. The viscous term on the right side of 2.4 serves as an entropy dissipative regularization (defined below) [12], making all discontinuities theoretically resolvable. The entropy solution satisfies

$$
q(x, t)=\lim _{\varepsilon \rightarrow 0} q^{\varepsilon}(x, t) .
$$

The entropy solution is so named because it satisfies the entropy condition, which for gas dynamics becomes a statement of the second law of thermodynamics. The general mathematical definition of entropy is a nonlinear scalar function, $S(q)$, with a corresponding entropy flux, $F(q)$, defined by the differential relation [13]

$$
S_{q} f_{q}=F_{q} .
$$

The mathematical entropy is convex, meaning that the Hessian is positive definite,

$$
\zeta^{T} S_{q q} \zeta>0, \quad \forall \zeta \neq 0,
$$

and yields a one-to-one mapping from conservation variables, $q$, to entropy variables, $w^{T}=S_{q}$. Premultiplying the regularized conservation law in 2.4 by the entropy variables yields the entropy equation,

$$
S_{q} q_{t}^{\varepsilon}+S_{q} f\left(q^{\varepsilon}\right)_{x}=S_{t}^{\varepsilon}+F_{x}^{\varepsilon}=\varepsilon S_{q} f_{x}^{(v)} .
$$


The viscous terms in 2.8 can be rewritten as

$$
\varepsilon S_{q} f_{x}^{(v)}=\varepsilon w^{T} f_{x}^{(v)}=\varepsilon\left(w^{T} f^{(v)}\right)_{x}-\varepsilon w_{x}^{T} f^{(v)},
$$

and because an entropy dissipative regularization [12] requires that

$$
w_{x}^{T} f^{(v)} \geq 0, \quad \forall w .
$$

then, an entropy dissipative regularization ensures that entropy is always dissipated by the viscous terms. Substituting the definition in 2.10 into 2.9 yields

$$
\varepsilon\left(w^{T} f^{(v)}\right)_{x} \geq \varepsilon w^{T}\left(f^{(v)}\right)_{x} .
$$

This relation is substituted into 2.8 to find the local entropy inequality [11],

$$
S_{t}^{\varepsilon}+F_{x}^{\varepsilon} \leq \varepsilon\left(w^{T} f^{(v)}\right)_{x} .
$$

The entropy condition for the conservation law is found by integrating 2.12 over space and taking the limit $\varepsilon \rightarrow 0$,

$$
\frac{d}{d t} \int_{x_{L}}^{x_{R}} S \mathrm{~d} x+\left.F\right|_{x_{L}} ^{x_{R}} \leq 0 .
$$

If the weak solution satisfies 2.13 , then it is the entropy solution consistent with the definition in 2.5. It is important to note that the mathematical entropy has the opposite sign from thermodynamic entropy in gas dynamics. Thus, the mathematical entropy across a shock decreases instead of increases. This nomenclature is used consistently throughout this document.

\subsection{Entropy Analysis}

The application of continuous entropy analysis was used above in the derivation of the entropy condition. Some additional formal definitions are useful to further specify the mathematical characteristics of the entropy.

As stated in Section 2.1, the mathematical entropy is a nonlinear function of the conservation variables, $S(q)$, with a corresponding nonlinear entropy flux, $F(q)$. A set of entropy variables, $w$, with a one-to-one mapping to the conservation variables, $q$, is defined based on this entropy. The entropy variables have some remarkable properties. Because of the one-to-one mapping, the conservative variables can be written as a function of the entropy variables, $q(w)$. Thus, the strong form of the conservation law can be rewritten as,

$$
q_{w} w_{t}+f_{q} q_{w} w_{x}=0 .
$$

Both $q_{w}$ and $f_{w}=f_{q} q_{w}$ are symmetric matrices [13], so the conservation law is a symmetric hyperbolic system when written in terms of entropy variables. 
In the development of the entropy condition, we assumed that the regularization terms were entropy dissipative, satisfying

$$
w_{x}^{T} f^{(v)} \geq 0, \quad \forall w .
$$

Following Hauke et al. [14] this is shown by casting the viscous flux in a quasi-linear form,

$$
f^{(v)}=c(q, x) q_{x},
$$

where $c(q, x)$ may be a scalar constant, a variable matrix that depends on spatial location, or nonlinear in the conservation variable. An entropy function can be chosen such that the viscous coefficients are symmetric and positive semi-definite,

$$
f^{(v)}=\hat{c} w_{x}, \quad \hat{c}=c(q, x) q_{w}, \quad \zeta^{T} \hat{c} \zeta^{T} \geq 0, \forall \zeta .
$$

It is clear that if this transformation holds, then

$$
w_{x}^{T} f^{(v)}=w_{x}^{T} \hat{c} w_{x} \geq 0, \quad \forall w,
$$

and the viscous regularization is indeed entropy dissipative. Other authors $[6,13,14]$ have noted that an entropy that makes the hyperbolic matrices symmetric will not necessarily make the diffusive coefficient matrix symmetric. Therefore, the space of possible entropy functions for a parabolic or incompletely parabolic system is reduced compared to the hyperbolic problem. This consideration is important when defining the entropy condition for a given problem. Herein we restrict our definition of entropy stability to entropy functions that satisfy a specific viscous regularization even when evaluating the stability for problems in the limit of zero viscosity.

For nonzero viscosity, the continuous entropy decay rate is found by substituting 2.16 into 2.8 and integrating over space,

$$
\frac{d}{d t} \int_{x_{L}}^{x_{R}} S \mathrm{~d} x=\left[\varepsilon w^{T} f^{(v)}-F\right]_{x_{L}}^{x_{R}}-\varepsilon \int_{x_{L}}^{x_{R}} w_{x}^{T} \hat{c} w_{x} \mathrm{~d} x .
$$

The last integral term is positive semi-definite and thus the entropy will only increase in the domain through the boundaries. The goal of the numerical methods designed in this paper will be to mimic 2.18 at the semi-discrete level.

Harten [13] describes that the symmetry of the matrices, $q_{w}$ and $f_{w}$, indicates that the conservation variables, $q$, and flux, $f$, are Jacobians of scalar functions with respect to the entropy variables,

$$
q^{T}=\varphi_{w}, \quad f^{T}=\psi_{w},
$$

where the nonlinear function, $\varphi$, is called the potential and $\psi$ is called the potential flux [6]. These nonlinear functions satisfy

$$
\varphi=w^{T} q-S, \quad \psi=w^{T} f-F .
$$

Just as the entropy function is convex with respect to the conservative variables ( $S_{q q}$ is positive definite), the potential function is convex with respect to the entropy 
variables. We note that the one-to-one mapping admits an alternate form of the flux based on the entropy variables, $g(w)=f(q)$.

The entropy and corresponding entropy flux are often referred to as an entropyentropy flux pair, $(S, F)$. Similarly, the potential and the corresponding potential flux are referred to as a potential-potential flux pair, $(\varphi, \psi)[6]$. The symmetry properties and the definition of the potential flux are used in the stability analyses in the rest of this work.

Entropy analysis is valid for nonlinear equations and discontinuous solutions. It is therefore more generally applicable than linear energy analysis and gives a stronger stability estimate. We now turn our attention to the mathematical formalism required in spatial discretizations in order to mimic the continuous entropy properties at the semi-discrete level.

\subsection{Spatial Discretization}

Most finite difference approximations rely on a uniform discretization of the domain in each direction. Typically this uniform discretization is conducted in a computational space, and then a transformation to a nonuniform physical space permits greater flexibility in approximating solutions with varying scales. In this work, we limit our attention to Cartesian domains and extend the results to curvilinear multi-block domains in the future.

An important element in the approach taken here is the use of complementary grids. These grids allow the finite difference operations to be written as simple flux differences, analogous to the approach of the finite volume method. In a previous paper [15], we showed that this telescopic flux difference form yields a generalized summation-by-parts (SBP) property that is used to show that the weak solution to 2.1 is recovered when the solution converges.

\subsubsection{Complementary Grids}

The domain $\Omega=\left[x_{L}, x_{R}\right]$ is divided into $(N-1)$ uniform segments with $N$ equispaced endpoints denoted by $\mathbf{x}$,

$$
\mathbf{x}=\left(x_{1}, x_{2}, \ldots, x_{N}\right)^{T}, \quad x_{i}=x_{L}+\frac{i-1}{N-1}\left(x_{R}-x_{L}\right), \quad i=1,2, \ldots N .
$$

Since the approximate solution is constructed at these points, they are referred to as solution points. It is useful to define a set of intermediate points prescribing bounding control volumes about each solution point. These $(N+1)$ points are referred to as flux points as they are similar in nature to the control volume edges employed in the finite volume method. The distribution of the flux points depends on the discretization operator. In standard second-order finite difference methods, the flux points are located half way between adjacent solution points. For higher-order finite differences, the flux points are located half way between solution points in the domain interior, but the spacing between flux points abruptly becomes nonuniform as the boundaries are approached to satisfy the summation-by-parts condition (explained below). The spacing between the flux points is incorporated into the finite 
difference operator using the norm, $\mathcal{P}$, where the diagonal elements of $\mathcal{P}$ are equal to the spacing between flux points,

$$
\begin{aligned}
& \overline{\mathbf{x}}=\left(\bar{x}_{0}, \bar{x}_{1}, \ldots \bar{x}_{N}\right)^{T}, \quad \bar{x}_{0}=x_{1}, \quad \bar{x}_{N}=x_{N}, \\
& \bar{x}_{i}-\bar{x}_{i-1}=\mathcal{P}_{(i)(i)}, \quad i=1,2, \ldots, N .
\end{aligned}
$$

In operator notation, this is equivalent to

$$
\Delta \overline{\mathbf{x}}=\mathcal{P} \mathbf{1}
$$

where

$$
\mathbf{1}=(1,1, \ldots, 1)^{T}
$$

is a vector with $N$ elements and

$$
\Delta=\left(\begin{array}{cccccc}
-1 & 1 & 0 & 0 & 0 & 0 \\
0 & -1 & 1 & 0 & 0 & 0 \\
0 & 0 & \ddots & \ddots & 0 & 0 \\
0 & 0 & 0 & -1 & 1 & 0 \\
0 & 0 & 0 & 0 & -1 & 1
\end{array}\right)
$$

is an $N \times(N+1)$ matrix that calculates the undivided difference of the two adjacent flux points evaluated at the solution point. Note that in 2.22 , the first and last flux points are the same as the first and last solution points. This consistency is needed to define unique operators. The discretization is illustrated in Figure 1.

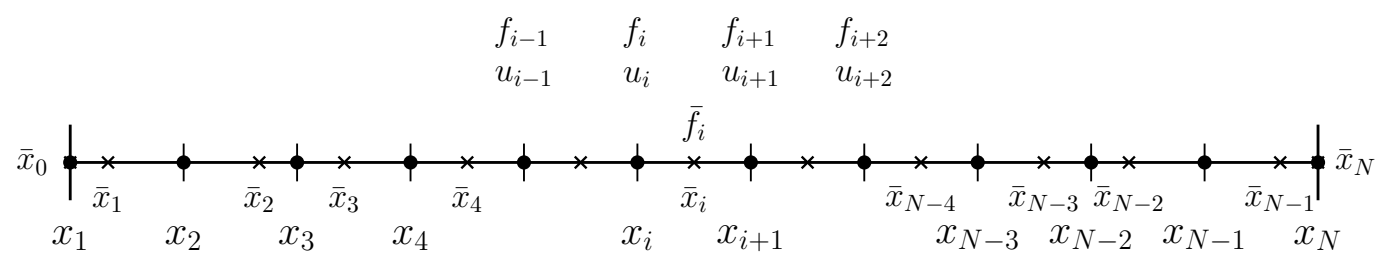

Figure 1. The one-dimensional discretization for finite differences is illustrated. Solution points are denoted by $\bullet$ and flux points are denoted by $\times$.

The approximate solution on the grid is denoted by

$$
\mathbf{u}(t)=\left(u_{1}(t), u_{2}(t), \ldots, u_{N}(t)\right)^{T}, \quad u_{i}(t)=u_{h}\left(x_{i}, t\right) \quad i=1,2, \ldots, N,
$$

where $q(x, t)$ has been reserved to denote exact solutions, and $u_{h}(x, t)$ denotes approximate solutions. Quantities located at flux points are denoted with an overbar.

Finite difference methods approximately satisfy the governing equation at the solution points, x. Difference methods based solely on the solutions points are considered next. These methods are then manipulated into simple flux differencing methods that use interpolated data at intermediate flux points. Recasting the methods in this way facilitates implementation as well as conservation properties. 


\subsubsection{First Derivative Approximation}

We utilize first derivative approximations that satisfy the summation-by-parts (SBP) condition, which means that the derivative approximation mimics integration-byparts,

$$
\int_{x_{L}}^{x_{R}} \phi u_{x} \mathrm{~d} x=\left.\phi u\right|_{x_{L}} ^{x_{R}}-\int_{x_{L}}^{x_{R}} \phi_{x} u \mathrm{~d} x .
$$

This mimetic property is achieved by constructing the first derivative approximation, $\mathcal{D} \phi$, with an operator in the form

$$
\begin{gathered}
\mathcal{D}=\mathcal{P}^{-1} \mathcal{Q}, \quad \mathcal{P}=\mathcal{P}^{T}, \quad \boldsymbol{\zeta}^{T} \mathcal{P} \boldsymbol{\zeta}>0, \quad \boldsymbol{\zeta} \neq \mathbf{0}, \\
\mathcal{Q}^{T}=\mathcal{B}-\mathcal{Q}, \quad \mathcal{B}=\operatorname{diag}(-1,0, \ldots, 0,1) .
\end{gathered}
$$

The function of the diagonal matrix $\mathcal{P}$ is to incorporate the local grid spacing into the derivative definition. The nearly skew-symmetric matrix, $\mathcal{Q}$, is an undivided differencing operator where all rows sum to zero and the first and last column sum to -1 and 1 , respectively. The difference operator, $\mathcal{D}$, approximates the first derivative as

$$
\phi_{x}(\mathbf{x})=\mathcal{D} \phi+\mathcal{T}_{p 2 p},
$$

where $\mathcal{T}_{p 2 p p}$ is the truncation error of the approximation. The nomenclature $2 p$ refers to the interior accuracy and $p$ refers to the accuracy at the left and right boundaries. Note that this truncation error $\left(\mathcal{T}_{p 2 p p}\right)$ is optimal if $\mathcal{D}$ is constrained to the diagonal norm case on a uniform grid and still satisfies the SBP property [16]. Integration in the approximation space is conducted using an inner product with the integration weights contained in the norm $\mathcal{P}$,

$$
\int_{x_{L}}^{x_{R}} \phi u_{x} \mathrm{~d} x \approx \phi^{T} \mathcal{P} \mathcal{D} \mathbf{u}, \quad \phi=\left(\phi\left(x_{1}\right), \phi\left(x_{2}\right), \ldots, \phi\left(x_{N}\right)\right)^{T} .
$$

Using the definition in 2.27, the SBP property is demonstrated,

$$
\phi^{T} \mathcal{P} \mathcal{P}^{-1} \mathcal{Q} \mathbf{u}=\phi^{T}\left(\mathcal{B}-\mathcal{Q}^{T}\right) \mathbf{u}=\phi_{N} u_{N}-\phi_{1} u_{1}-\phi^{\boldsymbol{T}} \mathcal{D}^{T} \mathcal{P} \mathbf{u}
$$

The specific operator used in this work is shown in Appendix A.1.

\subsubsection{Variable Coefficient Second Derivative Approximation}

The viscous approximations for regularized conservation laws, written in general as

$$
\left(\vartheta(x) v_{x}(\mathbf{x})\right)_{x}=\mathcal{D}_{2}(\vartheta) \mathbf{v}+\mathcal{T}_{p 2 p p}^{(v)},
$$

must also satisfy the SBP condition. Integration by parts yields

$$
\int_{x_{L}}^{x_{R}} \phi\left(\vartheta v_{x}\right)_{x} \mathrm{~d} x=\left.\phi \vartheta v_{x}\right|_{x_{L}} ^{x_{R}}-\int_{x_{L}}^{x_{R}} \phi_{x} \vartheta v_{x} \mathrm{~d} x .
$$


It is trivial to show that two applications of the first derivative operator satisfy the SBP condition. In practice, this is not advisable, as the approximation using two first derivative operations requires a much wider stencil (is less efficient), is less accurate, and leads to only neutrally stable approximations [17-19]. Instead the viscous operator is defined as

$$
\begin{gathered}
\mathcal{D}_{2}(\vartheta)=\mathcal{P}^{-1}(-\mathcal{M}(\vartheta)+\mathcal{B}[\vartheta] \mathcal{D}), \quad \mathcal{M}(\vartheta)=\mathcal{M}(\vartheta)^{T}, \quad[\vartheta]=\operatorname{diag}(\vartheta(\mathbf{x})), \\
\boldsymbol{\zeta}^{T} \mathcal{M}(\vartheta) \boldsymbol{\zeta} \geq 0, \quad \boldsymbol{\zeta}^{T}[\vartheta] \boldsymbol{\zeta} \geq 0, \quad \forall \boldsymbol{\zeta} .
\end{gathered}
$$

The inner product with the $\mathcal{P}$-norm yields

$$
\phi^{T} \mathcal{P} \mathcal{P}^{-1}(-\mathcal{M}(\vartheta)+\mathcal{B}[\vartheta] \mathcal{D}) \mathbf{v}=\phi^{T} \mathcal{B}[\vartheta] \mathcal{D} \mathbf{u}-\phi^{T} \mathcal{M}(\vartheta) \mathbf{v}
$$

It is clear that the boundary terms are mimicked from the continuous case, but based on the definition in 2.33 it is unclear that

$$
\int_{x_{L}}^{x_{R}} \phi_{x} \vartheta v_{x} \mathrm{~d} x \approx \phi^{T} \mathcal{M}(\vartheta) \mathbf{v} .
$$

The easiest way to show this is to restrict the definition in 2.33 to what Mattsson [19] calls compatible operators,

$$
\mathcal{M}(\vartheta)=\mathcal{D}^{T} \mathcal{P}[\vartheta] \mathcal{D}+\mathcal{R}(\vartheta), \quad \mathcal{R}(\vartheta)=\mathcal{R}(\vartheta)^{T}, \quad \boldsymbol{\zeta}^{T} \mathcal{R}(\vartheta) \boldsymbol{\zeta} \geq 0, \quad \forall \boldsymbol{\zeta}
$$

where $\mathcal{R}(\vartheta)$ is called the remainder matrix and scales with the grid spacing at the same order as the truncation error of the second derivative operator. This shows the relationship between applying two first derivative operators (called a wide stencil approximation) and directly applying the second derivative. $\mathcal{R}(\vartheta)$ is chosen such that the wider stencil terms in $\mathcal{D}^{T} \mathcal{P} \mathcal{D}$ vanish, but the approximation of the second derivative still holds. This is detailed below. The SBP condition is then demonstrated by

$$
\phi^{T} \mathcal{P} \mathcal{D}_{2}(\vartheta) \mathbf{v}=\phi^{T} \mathcal{B}[\vartheta] \mathcal{D} \mathbf{v}-\phi^{T} \mathcal{D}^{T} \mathcal{P}[\vartheta] \mathcal{D} \mathbf{v}-\phi^{T} \mathcal{R}(\vartheta) \mathbf{v}
$$

The last term is small and decreases quickly with increasing resolution. Therefore, the SBP property for the viscous term mimics integration by parts at the designed order of accuracy.

To calculate the remainder matrix, we alter slightly the approach taken by Mattsson [19],

$$
\mathcal{R}(\vartheta)=\sum_{k=1}^{n_{r}} \mathcal{N}_{k}^{T} \widetilde{[\vartheta]} \mathcal{N}_{k}, \quad \tilde{\boldsymbol{\zeta}}^{T}[\tilde{\vartheta}] \tilde{\boldsymbol{\zeta}} \geq 0, \quad \forall \tilde{\boldsymbol{\zeta}}
$$

where $\mathcal{N}_{k}$ are rectangular operators for higher derivatives and $[\tilde{\vartheta}]_{k}$ are diagonal matrices with a convex combination of the variable coefficients. The major difference between this approach and the approach originally proposed by Mattsson is the use of rectangular matrices, which simplifies the derivation somewhat. The derivation of the matrices for fourth-order is shown in Appendix A.2. 


\subsubsection{Telescopic Flux Form}

All flux gradient operations in this work are recast into a telescopic flux form,

$$
f_{x}(\mathbf{u})=\mathcal{P}^{-1} \Delta \overline{\mathbf{f}}+\mathcal{T}_{p 2 p p}
$$

This demonstrates the utility of the complementary grid as described in Section 2.3.1. We show in a previous paper [15] that various conservative and accurate flux gradients can be constructed in this manner with formal boundary closures based entirely on the SBP operator, $\mathcal{Q}$. This is extended to a general formula for entropy consistent finite differences in Section 3.4. When $\mathcal{Q}$ satisfies the SBP condition, the endpoint fluxes are always consistent,

$$
\bar{f}_{0}=f\left(u_{1}\right), \quad \bar{f}_{N}=f\left(u_{N}\right) .
$$

This telescopic flux form admits a generalized SBP property. The typical SBP operator in 2.27 transfers the action of the discrete derivative onto a test function with an equivalent order of approximation. The telescopic flux form combined with the flux consistency condition results in a more generalized relation,

$$
\phi^{T} \mathcal{P} \mathcal{P}^{-1} \Delta \overline{\mathbf{f}}=\phi^{T}(\tilde{\mathcal{B}}-\tilde{\Delta}) \overline{\mathbf{f}}=f\left(u_{N}\right) \phi_{N}-f\left(u_{1}\right) \phi_{1}-\phi \tilde{\Delta} \overline{\mathbf{f}},
$$

where

$$
\tilde{\Delta}=\left(\begin{array}{cccccc}
0 & -1 & 0 & 0 & 0 & 0 \\
0 & 1 & -1 & 0 & 0 & 0 \\
0 & 0 & \ddots & \ddots & 0 & 0 \\
0 & 0 & 0 & 1 & -1 & 0 \\
0 & 0 & 0 & 0 & 1 & 0
\end{array}\right), \quad \tilde{\mathcal{B}}=\left(\begin{array}{cccccc}
-1 & 0 & 0 & 0 & 0 & 0 \\
0 & 0 & 0 & 0 & 0 & 0 \\
0 & 0 & \ddots & \ddots & 0 & 0 \\
0 & 0 & 0 & 0 & 0 & 0 \\
0 & 0 & 0 & 0 & 0 & 1
\end{array}\right)
$$

and

$$
\frac{1}{\delta x} \phi^{T} \tilde{\Delta}=\phi_{x}^{T}+\mathcal{O}\left(N^{-1}\right)
$$

This is equivalent to the commonly used explanation of summation-by-parts in indicial form,

$$
\sum_{i=1}^{N} \phi_{i}\left(\bar{f}_{i}-\bar{f}_{i-1}\right)=f\left(u_{N}\right) \phi_{N}-f\left(u_{1}\right) \phi_{1}-\sum_{i=1}^{N-1} \bar{f}_{i}\left(\phi_{i+1}-\phi_{i}\right) .
$$

The action of the derivative is still moved onto the test function but at first order accuracy. This generalized property is important for satisfying the weak form, as shown in Section 2.4.

The variable coefficient viscous operators described in Section 2.3.3 can be constructed to satisfy

$$
\left(\vartheta v_{x}(\mathbf{x})\right)_{x} \approx \mathcal{P}^{-1}(-\mathcal{M}(\vartheta)+\mathcal{B}[\vartheta] \mathcal{D}) \mathbf{v}=\mathcal{P}^{-1} \Delta \overline{\mathbf{f}}^{(v)}
$$

This is shown by construction in Appendix A.2.1. 
Remark. The flux form is not commonly used in finite difference implementations, except by those that use WENO or hybrid WENO with central differencing. It is unnecessary to implement a scheme in this way, but it is important to be able to show that a scheme can be cast in this form. This was a critical step toward proving that different finite difference forms can be constructed to satisfy the Lax Wendroff theorem [15]. Furthermore, it is shown in Section 4.2.3 that sometimes the flux form is necessary to describe a difference operator when no simple differential form exists.

\subsubsection{Semi-Discretization}

The finite difference approximations described above are used to change the system of partial differential equations into a set of coupled ordinary differential equations. The result is the semi-discrete equation,

$$
\mathbf{u}_{t}+\mathcal{P}^{-1} \Delta \overline{\mathbf{f}}=\mathcal{P}^{-1} \Delta \overline{\mathbf{f}}^{(v)}+\mathcal{P}^{-1} \mathbf{g}_{b}
$$

where $\mathbf{g}_{b}$ contains the enforcement of boundary conditions, detailed in our previous work [15] and other references [20-24]. The algorithmic form,

$$
\left(u_{i}\right)_{t}=\frac{\left(\bar{f}_{i}^{(v)}-\bar{f}_{i-1}^{(v)}\right)-\left(\bar{f}_{i}-\bar{f}_{i-1}\right)+\left(g_{b}\right)_{i}}{\mathcal{P}_{(i)(i)}}, \quad i=1,2, \ldots, N
$$

is used in all applications in this work and illustrates how the complementary grids interact. Gradients are specified only using simple differences of interpolated fluxes at the flux points.

\subsection{Satisfying the Weak Form}

We showed in a previous paper [15] how the telescopic flux form described above can be used to guarantee that the weak solution is recovered when the solution converges. Two additional conditions are required [25]: All fluxes in $\overline{\mathbf{f}}$ must have compact support,

$$
\bar{f}_{j}=\bar{f}_{j}\left(u_{j-\ell+1}, u_{j-\ell+2}, \ldots, u_{j+\ell-1}, u_{j+\ell}\right), \quad j=0,1, \ldots, N
$$

where $\ell \geq 0$ is a finite integer independent of $N$; and $\overline{\mathbf{f}}$ must be consistent with the flux in the Rankine Hugoniot relation,

$$
\bar{f}_{j}(q, q, \ldots, q, q)=f(q)
$$

In other words, as the resolution increases, the flux stencil width must remain constant, and the functional form of the flux must match the functional form of the conservation law flux. 


\subsection{Temporal Integration}

In all simulations used herein, the low-storage, five-stage, fourth-order, Runge Kutta scheme of Carpenter and Kennedy [26] is used to advance the semi-discretization in time. It is noted that this scheme does not satisfy the strong stability preserving (SSP) property [27]. In the problems of interest for the current work, schemes that did satisfy the SSP property exhibited the same robustness and stability as the lowstorage scheme, so the extra cost associated with these methods was unnecessary. However, SSP Runge Kutta time integration and an appropriate limit on the time step are required for the formal proof to hold in time.

\section{$3 \quad$ Entropy Stable Finite Differences}

In this work, we seek spatial flux divergence approximations using the finite difference method that mimic the continuous entropy condition at the semi-discrete level. This is accomplished through a semi-discrete entropy analysis to determine the conditions on the telescopic flux form required to satisfy the mimetic property.

\subsection{Semi-Discrete Entropy Analysis}

The goal of the semi-discrete entropy analysis is to show that the semi-discrete entropy mimics a continuous entropy estimate such as that given in equation 2.18. The nonlinear analysis begins by contracting the entropy variables $\mathbf{w}^{T}$ with the semidiscrete equation 2.43. The resulting global equation that governs the semi-discrete decay of entropy is given by

$$
\mathbf{w}^{T} \mathcal{P} \mathbf{u}_{t}+\mathbf{w}^{T} \Delta \overline{\mathbf{f}}=\mathbf{w}^{T} \Delta \overline{\mathbf{f}}^{(v)}+\mathbf{w}^{T} \mathbf{g}_{b},
$$

where

$$
\mathbf{w}=\left(w\left(u_{1}\right)^{T}, w\left(u_{2}\right)^{T}, \ldots, w\left(u_{N}\right)^{T}\right)^{T},
$$

is the vector of entropy variables. The time derivative is easily manipulated into a mimetic form by using a diagonal norm SBP operator (which commutes with any arbitrary diagonal matrix) and the pointwise definition of entropy

$$
w_{i}^{T}\left(u_{i}\right)_{t}=\left(S_{i}\right)_{t}, \quad \forall i .
$$

The resulting expression is

$$
\mathbf{w}^{T} \mathcal{P} \mathbf{u}_{t}=\mathbf{1}^{T} \mathcal{P} \mathbf{S}_{t}
$$

Using this result, equation 3.1 is recast as

$$
\frac{d}{d t} \mathbf{1}^{T} \mathcal{P} \mathbf{S}=-\mathbf{w}^{T} \Delta\left(\overline{\mathbf{f}}-\overline{\mathbf{f}}^{(v)}\right)+\mathbf{w}^{T} \mathbf{g}_{b}
$$

This equation and mimetic arguments for semi-discrete entropy consistency will

yield sufficient conditions for $\overline{\mathbf{f}}, \overline{\mathbf{f}}^{(v)}$, and $\mathbf{g}_{b}$. The derivation of entropy consistent inviscid and viscous terms, however is considerably more involved than that required for the time term. 


\subsection{Inviscid Flux Conditions}

\subsubsection{Entropy Consistent Fluxes}

By definition, an entropy-consistent inviscid semi-discretization satisfies the expression

$$
\mathbf{w}^{T} \mathcal{P} \mathbf{u}_{t}+F\left(u_{N}\right)-F\left(u_{1}\right)=\frac{d}{d t} \mathbf{1}^{T} \mathcal{P} \mathbf{S}+F\left(u_{N}\right)-F\left(u_{1}\right)=\mathbf{w}^{T} \mathbf{g}_{b} .
$$

Comparing this expression with inviscid portion of equation 3.2 immediately reveals that the inviscid flux terms are entropy consistent if they satisfy

$$
\mathbf{w}^{T} \Delta \overline{\mathbf{f}}=F\left(u_{N}\right)-F\left(u_{1}\right)=\mathbf{1}^{T} \Delta \overline{\mathbf{F}} .
$$

It is difficult to enforce the global entropy consistency condition in 3.4 at the flux points. To circumvent this problem, Tadmor [6] developed a more restrictive condition that yields a local condition on the flux for the global entropy consistency. We generalize this condition in the current work for higher approximation orders and finite domains.

Substituting the definition for generalized summation-by-parts in Section 2.3.4, $\Delta=\tilde{\mathcal{B}}-\tilde{\Delta}$, into the global entropy consistency condition in 3.4 yields

$$
\mathbf{w}^{T} \tilde{\mathcal{B}} \overline{\mathbf{f}}-\mathbf{w}^{T} \tilde{\Delta} \overline{\mathbf{f}}-\mathbf{1}^{T} \tilde{\mathcal{B}} \overline{\mathbf{F}}+\mathbf{1}^{T} \tilde{\Delta} \overline{\mathbf{F}}=\mathbf{w}^{T} \tilde{\mathcal{B}} \overline{\mathbf{f}}-\mathbf{1}^{T} \tilde{\mathcal{B}} \overline{\mathbf{F}}-\mathbf{w}^{T} \tilde{\Delta} \overline{\mathbf{f}}=0 .
$$

The boundary terms in 3.5 can be reorganized as

$$
\mathbf{w}^{T} \tilde{\mathcal{B}} \overline{\mathbf{f}}-\mathbf{1}^{T} \tilde{\mathcal{B}} \overline{\mathbf{F}}=\left(w_{N}^{T} f_{N}-F_{N}\right)-\left(w_{1}^{T} f_{1}-F_{1}\right)=\psi_{N}-\psi_{1}=\tilde{\boldsymbol{\psi}}^{T} \tilde{\mathcal{B}} \overline{\mathbf{1}}
$$

where $\psi_{1}$ and $\psi_{N}$ represent the potential flux defined in 2.20 , and $\tilde{\boldsymbol{\psi}}$ is a vector of potential fluxes, discussed below. Defining $[\overline{\mathbf{f}}]$ as a diagonal $(N+1) \times(N+1)$ matrix containing the elements of $\overline{\mathbf{f}}, 3.4$ further simplifies to

$$
\left(\tilde{\boldsymbol{\psi}}^{T} \tilde{\mathcal{B}}-\mathbf{w}^{T} \tilde{\Delta}[\overline{\mathbf{f}}]\right) \overline{\mathbf{1}}=0 .
$$

Substituting the equality $\tilde{\boldsymbol{\psi}}^{T} \tilde{\mathcal{B}} \overline{\mathbf{1}}=\tilde{\boldsymbol{\psi}}^{T} \tilde{\Delta} \overline{\mathbf{1}}$ into the left side of the equation yields

$$
\left(\tilde{\boldsymbol{\psi}}^{T} \tilde{\Delta}-\mathbf{w}^{T} \tilde{\Delta}[\overline{\mathbf{f}}]\right) \overline{\mathbf{1}}=0 .
$$

This is satisfied by the vector sufficient condition,

$$
\tilde{\boldsymbol{\psi}}^{T} \tilde{\Delta}=\mathbf{w}^{T} \tilde{\Delta}[\overline{\mathbf{f}}], \quad \tilde{\psi}_{1}=\psi_{1}, \quad \tilde{\psi}_{N}=\psi_{N}
$$

and subsequently by the local sufficient conditions

$$
\left(w_{i+1}-w_{i}\right)^{T} \bar{f}_{i}=\tilde{\psi}_{i+1}-\tilde{\psi}_{i}, \quad i=1,2, \ldots, N-1 .
$$

A flux that satisfies this condition given in equation 3.8 is denoted $\overline{\mathbf{f}}^{(S)}$.

Note that Tadmor arrives at the condition $\tilde{\boldsymbol{\psi}}=\boldsymbol{\psi}$ because of an assumption on the form of $\overline{\mathbf{F}}[6]$. In the generalized condition derived in equation 3.7, it is 
unnecessary to define $\tilde{\psi}$ in the domain interior. Indeed, it is not even unique because of the arbitrary assumptions used to relate the matrices in the domain's interior. We shall see next that this generality is important for high-order methods, because the consistent entropy flux does not satisfy Tadmor's original form [7].

The following theorems are two important contribution of this work. They prove that high-order entropy consistent fluxes can be constructed from linear combinations of two-point entropy consistent fluxes. This results follows immediately from the structural properties of diagonal norm SBP operators, because they all admit the generalized SBP given in section 2.3.4.

Theorem 3.1. A two-point entropy consistent flux can be extended to high order with formal boundary closures using the form

$$
\bar{f}_{i}^{(S)}=\sum_{k=i+1}^{N} \sum_{\ell=1}^{i} 2 q_{(\ell, k)} \bar{f}_{S}\left(u_{\ell}, u_{k}\right), \quad 1 \leq i \leq N-1
$$

when the two-point non-dissipative function from Tadmor [6] is used

$$
\bar{f}_{S}\left(u_{k}, u_{\ell}\right)=\int_{0}^{1} g\left(w\left(u_{k}\right)+\xi\left(w\left(u_{\ell}\right)-w\left(u_{k}\right)\right)\right) \mathrm{d} \xi, \quad g(w(u))=f(u) .
$$

The coefficient $q_{(k, \ell)}$ corresponds to the $(k, \ell)$ row and column in $\mathcal{Q}$, respectively.

Proof. To show the accuracy of approximation, we express the flux difference as

$$
\bar{f}_{i}^{(S)}-\bar{f}_{i-1}^{(S)}=\sum_{k=i+1}^{N} \sum_{\ell=1}^{i} 2 q_{(\ell, k)} \bar{f}_{S}\left(u_{\ell}, u_{k}\right)-\sum_{k=i}^{N} \sum_{\ell=1}^{i-1} 2 q_{(\ell, k)} \bar{f}_{S}\left(u_{\ell}, u_{k}\right), \quad 2 \leq i \leq N-1 .
$$

The stencils of each flux contain considerable overlap, which is apparent if we rewrite the difference as

$$
\begin{aligned}
& \bar{f}_{i}^{(S)}-\bar{f}_{i-1}^{(S)}=\sum_{k=i+1}^{N} \sum_{\ell=1}^{i-1} 2 q_{(\ell, k)} \bar{f}_{S}\left(u_{\ell}, u_{k}\right)+\sum_{k=i+1}^{N} 2 q_{(i, k)} \bar{f}_{S}\left(u_{i}, u_{k}\right) \\
&-\sum_{k=i+1}^{N} \sum_{\ell=1}^{i-1} 2 q_{(\ell, k)} \bar{f}_{S}\left(u_{\ell}, u_{k}\right)-\sum_{\ell=1}^{i-1} 2 q_{(\ell, i)} \bar{f}_{S}\left(u_{\ell}, u_{i}\right), \\
&=\sum_{k=i+1}^{N} 2 q_{(i, k)} \bar{f}_{S}\left(u_{i}, u_{k}\right)-\sum_{\ell=1}^{i-1} 2 q_{(\ell, i)} \bar{f}_{S}\left(u_{\ell}, u_{i}\right), \\
& 2 \leq i \leq N-1 .
\end{aligned}
$$

Noting that $q_{(i, j)}=b_{(i, j)}-q_{(j, i)}$ and that $\bar{f}_{S}\left(u_{k}, u_{\ell}\right)=\bar{f}_{S}\left(u_{\ell}, u_{k}\right), 3.11$ is rewritten as

$$
\bar{f}_{i}^{(S)}-\bar{f}_{i-1}^{(S)}=\sum_{j=1}^{N} 2 q_{(i, j)} \bar{f}_{S}\left(u_{i}, u_{j}\right), \quad 2 \leq i \leq N-1 .
$$


By the same argument, the left boundary flux difference is

$$
\bar{f}_{1}^{(S)}-\bar{f}_{0}^{(S)}=\sum_{k=2}^{N} 2 q_{(1, k)} \bar{f}_{S}\left(u_{1}, u_{k}\right)-f\left(u_{1}\right)=\sum_{k=1}^{N} 2 q_{(1, k)} \bar{f}_{S}\left(u_{1}, u_{k}\right),
$$

and the right boundary difference is

$$
\bar{f}_{N}^{(S)}-\bar{f}_{N-1}^{(S)}=f\left(u_{N}\right)-\sum_{\ell=1}^{N-1} 2 q_{(\ell, N)} \bar{f}_{S}\left(u_{\ell}, u_{N}\right)=\sum_{k=1}^{N} 2 q_{(N, k)} \bar{f}_{S}\left(u_{N}, u_{k}\right) .
$$

Combining the expressions for the boundary points with 3.12 , the flux difference at all solution points is expressed as

$$
\bar{f}_{i}^{(S)}-\bar{f}_{i-1}^{(S)}=\sum_{j=1}^{N} 2 q_{(i, j)} \bar{f}_{S}\left(u_{i}, u_{j}\right), \quad 1 \leq i \leq N .
$$

This form facilitates an analysis by Taylor series at every solution point. The accuracy constraints of $\mathcal{Q}$ satisfy

$$
\sum_{k=1}^{N} \frac{q_{i k}(k-i)^{\ell}}{\ell !}=\frac{\mathcal{P}_{(i)(i)}}{\delta x} \delta_{1 \ell}, \quad \ell=0,1, \ldots, d,
$$

where $d=2 p$ in the domain interior and $d=p$ at the boundaries. To proceed further, we need to rewrite the flux function in 3.10 as a Taylor series expansion about $\xi=0[5]$,

$$
g\left(w\left(u_{i}\right)+\xi\left(w\left(u_{j}\right)-w\left(u_{i}\right)\right)\right)=\left.\sum_{k=0}^{\infty} \frac{1}{k !} \frac{\partial^{k} g}{\partial w^{k}}\right|_{w_{i}}\left(w_{j}-w_{i}\right)^{k} \xi^{k} .
$$

The integration now proceeds simply,

$$
\begin{aligned}
\bar{f}_{S}\left(u_{i}, u_{j}\right) & =\left.\int_{0}^{1} \sum_{k=0}^{\infty} \frac{1}{k !} \frac{\partial^{k} g}{\partial w^{k}}\right|_{w_{i}}\left(w_{j}-w_{i}\right)^{k} \xi^{k} \mathrm{~d} \xi \\
& =\left.\sum_{k=0}^{\infty} \frac{1}{k !} \frac{\partial^{k} g}{\partial w^{k}}\right|_{w_{i}}\left(w_{j}-w_{i}\right)^{k} \int_{0}^{1} \xi^{k} \mathrm{~d} \xi \\
& =\left.\sum_{k=0}^{\infty} \frac{1}{k !} \frac{\partial^{k} g}{\partial w^{k}}\right|_{w_{i}}\left(w_{j}-w_{i}\right)^{k} \frac{1}{k+1},
\end{aligned}
$$

and simplifies to

$$
\bar{f}_{S}\left(u_{i}, u_{j}\right)=g\left(w_{i}\right)+\left.\sum_{k=1}^{\infty} \frac{1}{(k+1) !} \frac{\partial^{k} g}{\partial w^{k}}\right|_{w_{i}}\left(w_{j}-w_{i}\right)^{k}
$$


We now perform a second Taylor expansion about $w_{i}$,

$$
\bar{f}_{S}\left(u_{i}, u_{j}\right)=g\left(w_{i}\right)+\left.\sum_{k=1}^{\infty} \frac{1}{(k+1) !} \frac{\partial^{k} g}{\partial w^{k}}\right|_{w_{i}}\left[\left.\sum_{\ell=1}^{\infty} \frac{(\delta x)^{\ell}(j-i)^{\ell}}{\ell !} \frac{\partial^{\ell} w}{\partial x^{\ell}}\right|_{x_{i}}\right]^{k} .
$$

This expression for the flux is substituted into 3.13,

$$
\bar{f}_{i}^{(S)}-\bar{f}_{i-1}^{(S)}=\sum_{j=1}^{N} 2 q_{i j}\left[g\left(w_{i}\right)+\left.\sum_{k=1}^{\infty} \frac{1}{(k+1) !} \frac{\partial^{k} g}{\partial w^{k}}\right|_{w_{i}}\left[\left.\sum_{\ell=1}^{\infty} \frac{(\delta x)^{\ell}(j-i)^{\ell}}{\ell !} \frac{\partial^{\ell} w}{\partial x^{\ell}}\right|_{x_{i}}\right]^{k}\right]
$$

The first term vanishes because all rows of $\mathcal{Q}$ sum to zero. We then split $k=1$ from the remaining terms in the sum,

$$
\begin{aligned}
\bar{f}_{i}^{(S)}-\bar{f}_{i-1}^{(S)} & =\left.\left.\sum_{j=1}^{N} \sum_{\ell=1}^{\infty} \frac{\partial g}{\partial w}\right|_{w_{i}} \frac{(\delta x)^{\ell} q_{i j}(j-i)^{\ell}}{\ell !} \frac{\partial^{\ell} w}{\partial x^{\ell}}\right|_{x_{i}} \\
& +\left.\sum_{j=1}^{N} \sum_{k=2}^{\infty} \frac{2 q_{i j}}{(k+1) !} \frac{\partial^{k} g}{\partial w^{k}}\right|_{w_{i}}\left[\left.\sum_{\ell=1}^{\infty} \frac{(\delta x)^{\ell}(j-i)^{\ell}}{\ell !} \frac{\partial^{\ell} w}{\partial x^{\ell}}\right|_{x_{i}}\right]^{k} .
\end{aligned}
$$

Using 3.14, the first term is rewritten as

$$
\left.\left.\sum_{j=1}^{N} \sum_{\ell=1}^{\infty} \frac{\partial g}{\partial w}\right|_{w_{i}} \frac{(\delta x)^{\ell} q_{i j}(j-i)^{\ell}}{\ell !} \frac{\partial^{\ell} w}{\partial x^{\ell}}\right|_{x_{i}}=\left.\left.\frac{\partial g}{\partial w}\right|_{w_{i}} \frac{\partial w}{\partial x}\right|_{x_{i}} \mathcal{P}_{(i)(i)}+\mathcal{O}\left((\delta x)^{d+1}\right),
$$

which will yield a design-order accurate approximation of $g_{x}=f_{x}$ at $x_{i}$. We now must show that the remaining sum term in 3.18 is design-order small. To handle the series raised to a power, we recursively apply the identity,

$$
\left(\sum_{k=1}^{\infty} a_{k} x^{k}\right)\left(\sum_{k=1}^{\infty} b_{k} x^{k}\right)=\left(\sum_{\ell_{1}=1}^{\infty} a_{\ell_{1}} x^{\ell_{1}}\right)\left(\sum_{\ell_{2}=1}^{\infty} b_{\ell_{2}} x^{\ell_{2}}\right)=\sum_{\ell_{1}=1}^{\infty} \sum_{\ell_{2}=1}^{\infty} a_{\ell_{1}} b_{\ell_{2}} x^{\left(\ell_{1}+\ell_{2}\right)},
$$

such that the expansion to the power $k$ can be rewritten as

$$
\left[\left.\sum_{\ell=1}^{\infty} \frac{(\delta x)^{\ell}(j-i)^{\ell}}{\ell !} \frac{\partial^{\ell} w}{\partial x^{\ell}}\right|_{x_{i}}\right]^{k}=\sum_{\ell_{1}=1}^{\infty} \cdots \sum_{\ell_{k}=1}^{\infty}\left(\prod_{m=1}^{k} \frac{\partial^{\ell_{m}} w}{\partial x^{\ell_{m}}}\right) \frac{(\delta x)^{\sum \ell_{m}}(j-i)^{\sum \ell_{m}}}{\left(\sum \ell_{m}\right) !} .
$$

Using

$$
\sum_{m=1}^{k} \ell_{m}>1, \quad \forall k \geq 2
$$

we can apply the accuracy constraints 3.14 to the second sum term in 3.18 ,

$$
\left.\sum_{j=1}^{N} \sum_{k=2}^{\infty} \frac{2 q_{i j}}{(k+1) !} \frac{\partial^{k} g}{\partial w^{k}}\right|_{w_{i}}\left[\left.\sum_{\ell=1}^{\infty} \frac{(\delta x)^{\ell}(j-i)^{\ell}}{\ell !} \frac{\partial^{\ell} w}{\partial x^{\ell}}\right|_{x_{i}}\right]^{k}=\mathcal{O}\left((\delta x)^{d+1}\right) .
$$


Thus we have shown that using 3.9,

$$
\frac{\bar{f}_{i}^{(S)}-\bar{f}_{i-1}^{(S)}}{\mathcal{P}_{(i)(i)}}=f_{x}+\mathcal{O}\left((\delta x)^{d}\right) .
$$

Theorem 3.2. A two-point high-order entropy consistent flux satisfying 3.8 with formal boundary closures can be constructed using 3.9,

$$
\bar{f}_{i}^{(S)}=\sum_{k=i+1}^{N} \sum_{\ell=1}^{i} 2 q_{(\ell, k)} \bar{f}_{S}\left(u_{\ell}, u_{k}\right), \quad 1 \leq i \leq N-1
$$

where $f_{S}\left(u_{\ell}, u_{k}\right)$ is any two-point non-dissipative function that satisfies the entropy consistency condition

$$
\left(w_{\ell}-w_{k}\right)^{T} \bar{f}_{S}\left(u_{\ell}, u_{k}\right)=\psi_{\ell}-\psi_{k}
$$

The high-order entropy consistent flux satisfies an additional local entropy consistency property,

$$
W \mathcal{P}^{-1} \Delta \overline{\mathbf{f}}^{(S)}=\mathcal{P}^{-1} \Delta \overline{\mathbf{F}}=F_{x}(\mathbf{u})+\mathcal{T}_{d}
$$

or equivalently,

$$
w_{i}^{T}\left(\bar{f}_{i}^{(S)}-\bar{f}_{i-1}^{(S)}\right)=\left(\bar{F}_{i}-\bar{F}_{i-1}\right), \quad 1 \leq i \leq N
$$

where

$$
\bar{F}_{i}=\sum_{k=i+1}^{N} \sum_{\ell=1}^{i} q_{(\ell, k)}\left[\left(w_{\ell}+w_{k}\right)^{T} \bar{f}_{S}\left(u_{\ell}, u_{k}\right)-\left(\psi_{\ell}+\psi_{k}\right)\right], \quad 1 \leq i \leq N-1 .
$$

Proof. Using 3.13, the inner product of the entropy variables with the flux difference can be expressed as

$$
\begin{aligned}
\mathbf{w}^{T} \Delta \overline{\mathbf{f}}^{(S)} & =\sum_{i=1}^{N} \sum_{j=1}^{N} 2 q_{(i, j)} w_{i}^{T} \bar{f}_{S}\left(u_{i}, u_{j}\right) \\
& =\sum_{i=1}^{N} \sum_{j=1}^{N}\left(q_{(i, j)}+b_{(i, j)}-q_{(j, i)}\right) w_{i}^{T} \bar{f}_{S}\left(u_{i}, u_{j}\right) .
\end{aligned}
$$

Using the structure of $\mathcal{B}$ and recognizing that the summation indices are arbitrary, and thus, $q_{(j, i)} w_{i}=q_{(i, j)} w_{j}$, we rewrite 3.24 as

$$
\mathbf{w}^{T} \Delta \overline{\mathbf{f}}^{(S)}=w_{N}^{T} f_{N}-w_{1}^{T} f_{1}+\sum_{i=1}^{N} \sum_{j=1}^{N} q_{(i, j)}\left(w_{i}-w_{j}\right)^{T} \bar{f}_{S}\left(u_{i}, u_{j}\right) .
$$


Tadmor [5] proved that

$$
\left(w_{\ell}-w_{k}\right)^{T} \int_{0}^{1} g\left(w\left(u_{k}\right)+\xi\left(w\left(u_{\ell}\right)-w\left(u_{k}\right)\right)\right) \mathrm{d} \xi=\psi_{\ell}-\psi_{k},
$$

but more generally we can show that any flux satisfying 3.20 can be used to simplify 3.25 to

$$
\begin{aligned}
\mathbf{w}^{T} \Delta \overline{\mathbf{f}}^{(S)} & =w_{N}^{T} f_{N}-w_{1}^{T} f_{1}+\sum_{i=1}^{N} \sum_{j=1}^{N} q_{(i, j)}\left(\psi_{i}-\psi_{j}\right) \\
& =w_{N}^{T} f_{N}-w_{1}^{T} f_{1}+\sum_{i=1}^{N} \psi_{i} \sum_{j=1}^{N} q_{(i, j)}-\sum_{i=1}^{N} \sum_{j=1}^{N} q_{(i, j)} \psi_{j} \\
& =F_{N}+\psi_{N}-F_{1}-\psi_{1}-\sum_{i=1}^{N} \sum_{j=1}^{N} q_{(i, j)} \psi_{j}=F_{N}-F_{1}
\end{aligned}
$$

where we have used $w^{T} f=F+\psi$. To the authors' knowledge, this proof of entropy consistency for high-order finite difference methods on bounded domains is new.

The accuracy proof of the local entropy consistency property 3.21 follows immediately from the accuracy proof of the flux difference 3.19,

$$
\begin{gathered}
\frac{w_{i}^{T}\left(\bar{f}_{i}^{(S)}-\bar{f}_{i-1}^{(S)}\right)}{\mathcal{P}_{(i)(i)}}=w_{i}^{T}\left(f_{x}\left(u_{i}\right)+\mathcal{O}\left((\delta x)^{d}\right)\right)=F_{x}\left(u_{i}\right)+\mathcal{O}\left((\delta x)^{d}\right) \\
1 \leq i \leq N .
\end{gathered}
$$

To show that 3.9 satisfies the local entropy consistency property 3.22 , we start by writing the entropy flux difference,

$$
\begin{gathered}
\bar{F}_{i}-\bar{F}_{i-1}=\sum_{k=i+1}^{N} \sum_{\ell=1}^{i} q_{(\ell, k)}\left[\left(w_{\ell}+w_{k}\right)^{T} \bar{f}_{S}\left(u_{\ell}, u_{k}\right)-\left(\psi_{\ell}+\psi_{k}\right)\right] \\
-\sum_{k=i}^{N} \sum_{\ell=1}^{i-1} q_{(\ell, k)}\left[\left(w_{\ell}+w_{k}\right)^{T} \bar{f}_{S}\left(u_{\ell}, u_{k}\right)-\left(\psi_{\ell}+\psi_{k}\right)\right] \\
2 \leq i \leq N-1 .
\end{gathered}
$$

Following the procedure in 3.11 to get 3.13 , we similarly get the simplified form of the entropy flux difference

$$
\bar{F}_{i}-\bar{F}_{i-1}=\sum_{j=1}^{N} q_{(i, j)}\left[\left(w_{i}+w_{j}\right)^{T} \bar{f}_{S}\left(u_{i}, u_{j}\right)-\left(\psi_{i}+\psi_{j}\right)\right], \quad 1 \leq i \leq N .
$$

To show the consistency, we premultiply 3.13 by $w_{i}^{T}$,

$$
w_{i}^{T}\left(\bar{f}_{i}^{(S)}-\bar{f}_{i-1}^{(S)}\right)=\sum_{j=1}^{N} 2 q_{(i, j)} w_{i}^{T} \bar{f}_{S}\left(u_{i}, u_{j}\right), \quad 1 \leq i \leq N .
$$


Adding and subtracting

$$
\sum_{j=1}^{N} q_{(i, j)} w_{j}^{T} \bar{f}_{S}\left(u_{i}, u_{j}\right)
$$

and using 3.20 , we can rewrite the difference as

$$
\begin{aligned}
w_{i}^{T}\left(\bar{f}_{i}^{(S)}-\bar{f}_{i-1}^{(S)}\right) & =\sum_{j=1}^{N} q_{(i, j)}\left[\left(w_{i}+w_{j}\right)^{T} \bar{f}_{S}\left(u_{i}, u_{j}\right)+\left(w_{i}-w_{j}\right)^{T} \bar{f}_{S}\left(u_{i}, u_{j}\right)\right] \\
& =\sum_{j=1}^{N} q_{(i, j)}\left[\left(w_{i}+w_{j}\right)^{T} \bar{f}_{S}\left(u_{i}, u_{j}\right)+\left(\psi_{i}-\psi_{j}\right)\right] .
\end{aligned}
$$

We now simply add

$$
-\psi_{i} \sum_{j=1}^{N} 2 q_{(i, j)}=0
$$

to get the flux difference formula consistent with 3.29 ,

$$
\begin{gathered}
w_{i}^{T}\left(\bar{f}_{i}^{(S)}-\bar{f}_{i-1}^{(S)}\right)=\sum_{j=1}^{N} q_{(i, j)}\left[\left(w_{i}+w_{j}\right) \bar{f}_{S}\left(u_{i}, u_{j}\right)-\left(\psi_{i}+\psi_{j}\right)\right]=F_{i}-F_{i-1}, \\
1 \leq i \leq N .
\end{gathered}
$$

Using Theorems 3.1 and 3.2, we are guaranteed that the extension of the twopoint flux, 3.9, is a high-order accurate entropy consistent discretization of the conservation law.

Remark. The entropy consistency proof is satisfied for all two-point fluxes that satisfy 3.20. The accuracy proof has only been proven for fluxes in the integral form 3.10. We are at this point unable to show that any flux satisfying 3.20 will be design-order accurate, so such fluxes should be validated for accuracy independent of Theorem 3.1.

\subsubsection{Entropy Stability}

For entropy stability an analogous condition to 3.3 is

$$
\mathbf{w}^{T} \mathcal{P} \mathbf{u}_{t}+F\left(u_{N}\right)-F\left(u_{1}\right) \leq \mathbf{w}^{T} \mathbf{g}_{b} .
$$

We again substitute the semi-discrete conservation law into 3.31 and show that the entropy stable inviscid fluxes must satisfy

$$
\mathbf{w}^{T} \Delta \overline{\mathbf{f}} \geq \mathbf{1}^{T} \Delta \overline{\mathbf{F}} .
$$

Using the result for the entropy consistent flux in 3.4, this condition can be rewritten as

$$
\mathbf{w}^{T} \Delta \overline{\mathbf{f}} \geq \mathbf{w}^{T} \Delta \overline{\mathbf{f}}^{(S)} .
$$


Substituting the generalized summation-by-parts property,

$$
\mathbf{w}^{T}(\tilde{\mathcal{B}}-\tilde{\Delta})\left(\overline{\mathbf{f}}^{(S)}-\overline{\mathbf{f}}\right)=\mathbf{w}^{T} \tilde{\Delta}\left(\overline{\mathbf{f}}-\overline{\mathbf{f}}^{(S)}\right)=\mathbf{w}^{T} \tilde{\Delta}\left([\overline{\mathbf{f}}]-\left[\overline{\mathbf{f}}^{(S)}\right]\right) \overline{\mathbf{1}} \leq 0,
$$

the sufficient local condition for entropy stability is

$$
\mathbf{w}^{T} \tilde{\Delta}[\overline{\mathbf{f}}] \leq \mathbf{w}^{T} \tilde{\Delta}\left[\overline{\mathbf{f}}^{(S)}\right],
$$

or in indicial form,

$$
\left(w_{i+1}-w_{i}\right)^{T}\left(\bar{f}_{i}-\bar{f}_{i}^{(S)}\right) \leq 0, \quad i=1,2, \ldots, N-1 .
$$

The development of this condition relied heavily on the formalism introduced in Section 2.3. The generalized SBP property made it possible to extend the entropy stability condition to finite domains.

Remark. The entropy stability condition is based on the global property 3.32. It has not been proved to provide a pointwise entropy stability property at this point.

The entropy stability condition in 3.35 can be used to find entropy stable conditions for any type of telescopic flux operator. It only informs the upper limit of dissipation from a flux operator such that the entropy condition is satisfied. It does not inform how to add sufficient dissipation such that a non-oscillatory solution is obtained. In this work, we use the WENO finite difference method to construct dissipation and use the condition 3.35 to ensure that the discretization is entropy stable. This is detailed in the following section.

\subsection{Entropy Stable WENO Finite Differences}

It is well known that non-dissipative numerical methods cannot be used to simulate shocks. The primary reason for this is that shocks dissipate energy and nondissipative numerical methods have no mechanism to mimic this. To simulate problems with shocks, dissipation needs to be added to the numerical method. There are a variety of mechanisms to achieve this, but in this work WENO finite difference methods are used. The implementation uses unique formal boundary closures from Fisher et al. [10] that satisfy the SBP condition. Stencil biasing mechanics follow two papers by Yamaleev and Carpenter $[28,29]$. The details of the generally applicable correction procedure are detailed below. The full implementation details including the WENO stencil biasing algorithm throughout the domain are available in Fisher et al. [10] and Carpenter et al. [30] for (2-4-2) and (3-6-3) operators, respectively. ${ }^{1}$

The first step to construct a WENO finite difference operator is to cast the difference operator in flux form,

$$
\mathcal{Q} \mathbf{f}=\Delta \overline{\mathbf{f}} .
$$

These fluxes that recover the non-dissipative first derivative approximation are called target fluxes. The target fluxes are broken into a sum of fluxes on smaller stencils

\footnotetext{
${ }^{1}$ The nomenclature (2-4-2) signifies that boundaries/interior stencils are second- and fourthorder accurate, respectively. The resulting operators are globally third- and fourth-order accurate, respectively.
} 
of width, $p$, called candidate stencils,

$$
\bar{f}_{j}=\sum_{k=1}^{n_{s}} \bar{d}_{j}^{k} \bar{f}_{j}^{S_{k}}, \quad j=1,2, \ldots, N-1,
$$

where $n_{s}$ is the number of candidate stencils needed to describe the target flux, $\bar{f}_{j}^{S_{k}}$ are the candidate fluxes, and $\bar{d}_{j}^{k}$ are the target weights that recover the target flux. The candidate stencil width is held constant for all fluxes in the domain. For example, fourth-order operators use $p=2$, and sixth-order operators use $p=3$. Figure 2 shows the fourth-order case. The number of candidate stencils needed to describe the fluxes $\bar{f}_{j}$ can vary, as the target fluxes do not all have the same stencil size when approaching the boundary. The functional form of the candidate stencils depends on the distribution of the flux points, and thus is fully described by the norm, $\mathcal{P}$, and the desired order of accuracy.

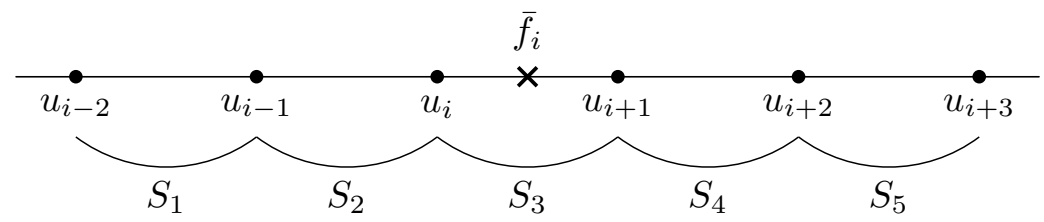

Figure 2. The stencil for a WENO scheme with $p=2$ and $n_{s}=5$ candidate stencils is shown.

WENO works by preventing the interpolated fluxes, $\bar{f}_{j}$, from using data across discontinuities. This is done by replacing the target weights, $\bar{d}_{j}^{k}$ with nonlinear weights,

$$
\bar{\omega}_{j}^{k}=\frac{\bar{\alpha}_{j}^{k}}{\sum_{\ell} \bar{\alpha}_{j}^{\ell}}, \quad \bar{\alpha}_{j}^{k}=\bar{d}_{j}^{k}\left(1+\frac{\bar{\tau}_{j}}{\bar{\beta}_{j}^{k}+\bar{\epsilon}_{j}}\right), \quad k=1, \ldots, n_{s} .
$$

The functional form of the nonlinear weights relies on the scaling parameter, $\bar{\epsilon}$, and dual stencil-biasing parameters, $\bar{\tau}$ and $\bar{\beta}$. $\bar{\tau}$ is a measure of the smoothness over the full stencil,

$$
\bar{\tau}_{j}=\sum_{i=1}^{n_{\tau}}\left(\frac{\partial^{2 p-1} u\left(\bar{x}_{j}\right)}{\partial x^{2 p-1}}(\delta x)^{2 p-1}\right)^{2}, \quad n_{\tau}=n_{s}-p .
$$

$\bar{\beta}$ is a measure of the smoothness over each individual candidate stencil,

$$
\bar{\beta}_{j}^{k}=\sum_{\ell=1}^{p-1}(\delta x)^{2 \ell}\left(\frac{\partial^{\ell} \varphi_{j}^{k}\left(\bar{x}_{j}\right)}{\partial x^{\ell}}\right)^{2}
$$

where $\varphi_{j}^{k}(x)$ is the unique order $(p-1)$ polynomial fit of the solution over the candidate stencil, $S_{k}$. The flux of the WENO scheme is calculated using the formula

$$
\bar{f}_{j}^{(W)}=\sum_{k=1}^{n_{s}} \bar{\omega}_{j}^{k} \bar{f}_{j}^{S_{k}}, \quad j=1,2, \ldots, N-1 .
$$


The stencil biasing procedure ensures that stencils containing discontinuities result in high relative values of $\bar{\beta}$ and are assigned negligible weights. The WENO flux becomes an interpolation that incorporates only smooth data. Away from discontinuities, the flux collapses to the target flux. Near discontinuities, the flux transforms into an upwind operator. Note that the flux consistency condition on the first and last flux point in 2.39 is enforced.

The WENO method as described has no provable stability properties. We employ a limiting procedure to ensure that it satisfies entropy stability. From 3.35, we require that

$$
\left(w_{i+1}-w_{i}\right)^{T}\left(\bar{f}_{i}^{(S S W)}-\bar{f}_{i}^{(S)}\right) \leq 0,
$$

where $f^{(S S W)}$ is the entropy stable WENO flux and $f^{(S)}$ is the entropy consistent flux. The limiter that guarantees entropy stability is chosen as

$$
\begin{gathered}
\bar{f}_{i}^{(S S W)}=\bar{f}_{i}^{(W)}+\delta\left(\bar{f}_{i}^{(S)}-\bar{f}_{i}^{(W)}\right), \quad \delta=\frac{\sqrt{b^{2}+c^{2}}-b}{\sqrt{b^{2}+c^{2}}}, \\
b=\left(w_{i+1}-w_{i}\right)^{T}\left(\bar{f}_{i}^{(S)}-\bar{f}_{i}^{(W)}\right), \quad c=10^{-12},
\end{gathered}
$$

where $f_{i}^{(W)}$ is the WENO flux without the correction described above. The limiting process to get the entropy stable property is not unique. It was chosen merely because it is smooth with respect to the solution. This same limiting procedure works with fluxes other than WENO. It is shown in a later section that this minor correction to the WENO operator has a large impact.

Remark. While this limiting process can be used on other dissipative methods to ensure the entropy stability property, we note that the selected dissipative method must be cast into telescopic-flux form and satisfy the generalized summation-byparts property. In other words, the dissipative part of the method must have formal boundary closures and satisfy the entropy consistency condition.

\subsection{Entropy Stable Viscous Terms}

Using the formalism introduced in Section 2.3.3 combined with the definition of the entropy dissipative regularization in Section 2.2, we can define viscous terms such that the continuous entropy properties are mimicked by the semi-discrete equation. This is accomplished simply by requiring that the discrete viscous fluxes are written in dependence on the discrete gradients of the entropy variables,

$$
\begin{gathered}
\left(\hat{c} w_{x}\right)_{x}=\mathcal{P}^{-1} \Delta \overline{\mathbf{f}}^{(v)}=\mathcal{D}_{2}(\hat{c}) \mathbf{w}+\mathcal{T}_{p 2 p}, \\
\mathcal{D}_{2}(\hat{c}) \mathbf{w}=\mathcal{P}^{-1}(-\mathcal{M}(\hat{c})+\mathcal{B}[\hat{c}] \mathcal{D}) \mathbf{w} \\
\mathcal{M}(\hat{c})=\mathcal{D}^{T} \mathcal{P}[\hat{c}] \mathcal{D}+\mathcal{R}(\hat{c}), \quad \mathcal{R}(\hat{c})=\sum_{k=1}^{n_{r}} \mathcal{N}_{k}^{T}[\widetilde{\hat{c}}]_{k} \mathcal{N}_{k} .
\end{gathered}
$$

The accuracy requirements are automatically satisfied. The coefficient matrices $[\hat{c}]$ and $[\widetilde{\hat{c}}]$ are positive semi-definite because they are constructed using block-diagonal combinations of positive semi-definite matrices. 
The contribution of the viscous terms to the semi-discrete entropy decay rate is

$$
\mathbf{w}^{T} \Delta \overline{\mathbf{f}}^{(v)}=\mathbf{w} \mathcal{B}[\hat{c}] \mathcal{D} \mathbf{w}-(\mathcal{D} \mathbf{w})^{T} \mathcal{P}[\hat{c}](\mathcal{D} \mathbf{w})-\sum_{k=1}^{n_{r}}\left(\mathcal{N}_{k} \mathbf{w}\right)^{T}[\tilde{c}]_{k}\left(\mathcal{N}_{k} \mathbf{w}\right)
$$

where the last term is design-order small, and the last two terms are negative semidefinite. Note that no definite properties can be defined if the discrete viscous fluxes are constructed based on gradients of primitive or conservative variables. While at the continuous level, $w_{x}=w_{q} q_{x}$, at the discrete level in general we must assume $\mathcal{D} \mathbf{w} \neq \mathbf{w}_{\mathbf{u}} \mathcal{D} \mathbf{u}$. As in 2.18, only the boundary term will result in growth of the entropy, and thus this approximation of the viscous terms is entropy stable.

This formal definition of high-order entropy stable viscous terms with full boundary closures appears to be completely new (to our knowledge). It can be used along with the entropy consistent inviscid terms to construct a semi-discretization that mimics the properties of the continuous entropy, which is shown in the next section.

\subsection{Entropy Stable Semi-Discretization}

We substitute the entropy stable fluxes for the inviscid 3.42 and viscous 3.43 terms into the semi-discrete conservation law 2.43 to get what we define as an entropy stable semi-discretization on the finite domain,

$$
\mathbf{u}_{t}+\mathcal{P}^{-1} \Delta \overline{\mathbf{f}}^{(S S W)}=\mathcal{P}^{-1} \Delta \overline{\mathbf{f}}^{(v)}+\mathcal{P}^{-1} \mathbf{g}_{b}
$$

Note that the boundary conditions imposed with the penalty $\mathbf{g}_{b}$ have not been considered. This is because we have found it necessary to specify the conservation law and the entropy-entropy flux pair to derive appropriate boundary conditions. In the case of the compressible Navier-Stokes equations, the continuous theory for entropy stable boundary conditions is lacking, and no satisfactory entropy stable boundary conditions have been derived. Instead, linearly stable boundary conditions are used in the penalties. The particular boundary conditions used for each conservation law are addressed in the next sections.

\section{Applications}

The new methodology developed herein is applied to two sets of conservation laws. Developments in Burgers equation are used to illustrate how a fully entropy stable scheme is constructed. The methodology is also extended to three dimensions for the compressible Euler and Navier-Stokes equations, which are the target application for this work. As mentioned above, full entropy stability cannot be achieved because of incomplete continuous theory at the boundaries. The entropy stable numerics are applied where possible. 


\subsection{Burgers Equation}

Burgers equation is a nonlinear model problem that admits shocks in the inviscid limit,

$$
\begin{gathered}
q_{t}+f(u)_{x}=\varepsilon f_{x}^{(v)}, \quad f(q)=\frac{q^{2}}{2}, \quad f^{(v)}=q_{x}, \quad x \in\left[x_{L}, x_{R}\right], \quad t \in[0, \infty), \\
\frac{q\left(x_{L}, t\right)+\left|q\left(x_{L}, t\right)\right|}{3} q\left(x_{L}, t\right)-\varepsilon q_{x}\left(x_{L}, t\right)-g_{L}(t)=0, \\
\frac{q\left(x_{R}, t\right)-\left|q\left(x_{R}, t\right)\right|}{3} q\left(x_{R}, t\right)-\varepsilon q_{x}\left(x_{R}, t\right)+g_{R}(t)=0, \\
q(x, 0)=g_{0}(x) .
\end{gathered}
$$

The boundary conditions in 4.1 are constructed such that the entropy corresponding to

$$
(S, F)=\left(\frac{q^{2}}{2}, \frac{q^{3}}{3}\right), \quad(\varphi, \psi)=\left(\frac{q^{2}}{2}, \frac{q^{3}}{6}\right),
$$

only increases with respect to the imposed data and maintains the same form in the inviscid limit $\varepsilon \rightarrow 0$. The entropy variables are $w=q$. We substitute this definition into the entropy decay rate 2.18 and find

$$
\frac{d}{d t} \int_{x_{L}}^{x_{R}} \frac{q^{2}}{2} \mathrm{~d} x=\left[\varepsilon q q_{x}-\frac{q^{3}}{3}\right]_{x_{L}}^{x_{R}}-\varepsilon \int_{x_{L}}^{x_{R}} q_{x} q_{x} \mathrm{~d} x .
$$

Using the boundary condition in 4.1 to replace $\varepsilon q_{x}$ at the boundaries above, the entropy decay rate becomes

$$
\frac{d}{d t} \int_{x_{L}}^{x_{R}} S \mathrm{~d} x=-\frac{\left|q_{R}\right|}{3} q_{R}^{2}-\frac{\left|q_{L}\right|}{3} q_{L}^{2}+q_{R} g_{R}+q_{L} g_{L}-\varepsilon \int_{x_{L}}^{x_{R}} q_{x}^{2} \mathrm{~d} x
$$

where $q_{L}=q\left(x_{L}, t\right)$ and $q_{R}=q\left(x_{R}, t\right)$. It is immediately clear that when $q_{L}=0$ or $q_{R}=0$, the corresponding boundary terms do not contribute to the entropy decay. When $q_{L} \neq 0$ or $q_{R} \neq 0$, the identity,

$$
y z=-\frac{1}{2}\left(\sqrt{a} y-\frac{1}{\sqrt{a}} z\right)^{2}+\frac{a}{2} y^{2}+\frac{1}{2 a} z^{2}, \quad a>0,
$$

is used to transform the corresponding positive terms in 4.4, which yields the entropy decay rate

$$
\begin{aligned}
\frac{d}{d t} \int_{x_{L}}^{x_{R}} S \mathrm{~d} x= & -\varepsilon \int_{x_{L}}^{x_{R}} q_{x}^{2} \mathrm{~d} x \\
& -\frac{1}{2}\left(\sqrt{a_{R}} q_{R}-\frac{1}{\sqrt{a_{R}}} g_{R}\right)^{2}-\frac{1}{2}\left(\sqrt{a_{L}} q_{L}-\frac{1}{\sqrt{a_{L}}} g_{L}\right)^{2} \\
& +\left(\frac{a_{R}}{2}-\frac{\left|q_{R}\right|}{3}\right) q_{R}^{2}+\left(\frac{a_{L}}{2}-\frac{\left|q_{L}\right|}{3}\right) q_{L}^{2}+\frac{1}{2 a_{R}} g_{R}^{2}+\frac{1}{2 a_{L}} g_{L}^{2} .
\end{aligned}
$$


The coefficients used to complete the square, $a_{L}$ and $a_{R}$, are arbitrary, and thus it is acceptable to set them to values that satisfy the proof,

$$
0<a_{R} \leq \frac{2}{3}\left|q_{R}\right|, \quad 0<a_{L} \leq \frac{2}{3}\left|q_{L}\right| .
$$

This shows that the only terms in 4.6 that will contribute to growth of the entropy are the boundary data terms, $\frac{1}{2 a_{L}} g_{L}^{2}$ and $\frac{1}{2 a_{R}} g_{R}^{2}$. This result is independent of the value of $\varepsilon$.

\subsubsection{Entropy Stable Discretization of Burgers Equation}

The inviscid terms in the discretization of Burgers equation are specified according to $3.42,3.9$, and 3.10 , where the two-point entropy consistent flux simplifies to

$$
\bar{f}_{S}\left(u_{k}, u_{\ell}\right)=\frac{1}{6}\left(u_{k} u_{k}+u_{k} u_{\ell}+u_{\ell} u_{\ell}\right) .
$$

The viscous terms are approximated according to 3.43 with the recognition that $\hat{c}=1$. For Burgers equation, entropy stable boundary penalties can be constructed, which are equivalent to the penalties derived in [15],

$$
\begin{aligned}
\mathbf{g}_{b}= & -\mathbf{e}_{1}\left(\frac{u_{1}+\left|u_{1}\right|}{3} u_{1}-\varepsilon(D \mathbf{u})_{1}-g_{L}\right) \\
& +\mathbf{e}_{N}\left(\frac{u_{N}-\left|u_{N}\right|}{3} u_{N}-\varepsilon(D \mathbf{u})_{N}+g_{R}\right) .
\end{aligned}
$$

The $\mathbf{e}$ vectors ensure that only the first and last elements of $\mathbf{g}_{b}$ are nonzero. The contribution of the penalties to the discrete entropy is

$$
\begin{aligned}
\mathbf{u}^{T} \mathbf{g}_{b}= & -\left(\frac{u_{1}+\left|u_{1}\right|}{3} u_{1}^{2}-\varepsilon u_{1}(D \mathbf{u})_{1}-u_{1} g_{L}\right) \\
& +\left(\frac{u_{N}-\left|u_{N}\right|}{3} u_{N}^{2}-\varepsilon u_{N}(D \mathbf{u})_{N}+u_{N} g_{R}\right) .
\end{aligned}
$$

Note that the form of the penalties matches the boundary condition specification in 4.1. The individual discrete elements are combined using 3.45. The semi-discrete entropy decay rate is

$$
\begin{aligned}
\frac{d}{d t} \mathbf{u}^{T} \mathcal{P} \mathbf{u} \leq & -\frac{1}{3}\left(u_{N}^{3}-u_{1}^{3}\right)+\frac{1}{3}\left(u_{N}^{3}-u_{1}^{3}-\left|u_{N}\right| u_{N}^{2}-\left|u_{1}\right| u_{1}^{2}\right) \\
& -\varepsilon \mathbf{u}^{T} \mathcal{M} \mathbf{u}+u_{1} g_{L}+u_{N} g_{R} .
\end{aligned}
$$

Using the relation in 4.5 to transform the positive terms yields the entropy expression

$$
\begin{aligned}
& \frac{d}{d t} \mathbf{1}^{T} \mathcal{P} \mathbf{S} \leq-\varepsilon \mathbf{u}^{T} \mathcal{M} \mathbf{u}+\frac{1}{2 a_{L}} g_{L}^{2}+\frac{1}{2 a_{R}} g_{R}^{2}
\end{aligned}
$$

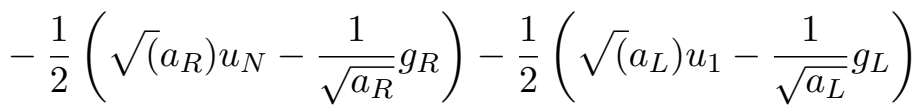

$$
\begin{aligned}
& +\left(\frac{a_{R}}{2}-\frac{\left|u_{N}\right|}{3}\right) u_{N}^{2}+\left(\frac{a_{L}}{2}-\frac{\left|u_{1}\right|}{3}\right) u_{1}^{2}, \\
& 0<a_{L} \leq \frac{2}{3}\left|u_{1}\right|, \quad 0<a_{R} \leq \frac{2}{3}\left|u_{N}\right| .
\end{aligned}
$$


Just as in 4.6 , the only terms that lead to entropy growth are $\frac{1}{2 a_{L}} g_{L}^{2}$ and $\frac{1}{2 a_{R}} g_{R}^{2}$. This ensures that the semi-discrete solution will remain bounded as long as appropriate boundary data are used.

\subsubsection{Advantages of Entropy Stability}

We show in this section how schemes designed for linear stability may fail at shocks and illustrate how entropy stable schemes can overcome such deficiencies. A linearlystable WENO scheme, termed ESWENO, was developed in Fisher et al. [10] with full SBP boundary closures. This scheme is constructed such that when the WENO operator is cast in flux form,

$$
\Delta \overline{\mathbf{f}}^{(E S W)}=(\tilde{\mathcal{Q}}+\tilde{\mathcal{R}}) \mathbf{f}, \quad \tilde{\mathcal{Q}}+\tilde{\mathcal{Q}}^{T}=\mathcal{B}, \quad \mathcal{R}=\mathcal{R}^{T}
$$

the symmetric dissipation matrix $\mathcal{R}$ is positive or negative semi-definite for waves with positive or negative propagation speeds, respectively. In comparison with the base WENO operator used in this work, the ESWENO operator incorporates an additional correction matrix,

$$
\Delta \overline{\mathbf{f}}^{(E S W)}-\Delta \overline{\mathbf{f}}^{(W)}=\mathcal{R}_{c} \mathbf{f},
$$

where $\mathcal{R}_{c}$ is a symmetric semi-definite matrix. This correction matrix has been shown to be necessary in linear equations where the one-sided stencil biasing mechanics become poorly behaved if no correction is added and result in solutions that do not converge. However, it has been observed that the ESWENO scheme exhibits overshoots at stationary shocks, which is not surprising because it is based on linear theory. This is illustrated by recognizing that the contribution of the energy correction term to the nonlinear entropy is

$$
\mathbf{u}^{T}\left(\Delta \overline{\mathbf{f}}^{(E S W)}-\Delta \overline{\mathbf{f}}^{(W)}\right)=\mathbf{u}^{T} \mathcal{R}_{c} \mathbf{f}=\frac{1}{2} \mathbf{u}^{T} \mathcal{R}_{c} U \mathbf{u}, \quad U=\operatorname{diag}(\mathbf{u}) .
$$

The ESWENO procedure ensures that $\mathcal{R}_{c}$ is semi-definite, but the matrix $\mathcal{R}_{c} U$ may be indefinite and thus may contribute to entropy growth. The disconnect between the intended behavior and the actual behavior of the correction term illustrates the shortfalls of linear analysis. Instead, if the entropy stable WENO (SSWENO) scheme developed herein is applied, the WENO scheme will only dissipate entropy.

The above behavior is illustrated using a common example defined by

$$
\begin{gathered}
x_{L}=-1, \quad x_{R}=1, \quad \varepsilon=0, \quad t \in[0,2.5], \\
g_{L}(t)=1, \quad g_{R}(t)=-1, \quad g_{0}(x)=-x .
\end{gathered}
$$

This problem is discretized using $N=65$ points. Figure 3 compares the simulation results at $t=2.5$ obtained using the WENO, ESWENO, and SSWENO schemes. Clearly, the linearly stable ESWENO operator exhibits a dramatic overshoot at the shock, while the WENO and SSWENO schemes are monotone.

While the WENO solution may appear to be the best solution, that is not in general what is observed. The SSWENO scheme exhibits better stability properties 


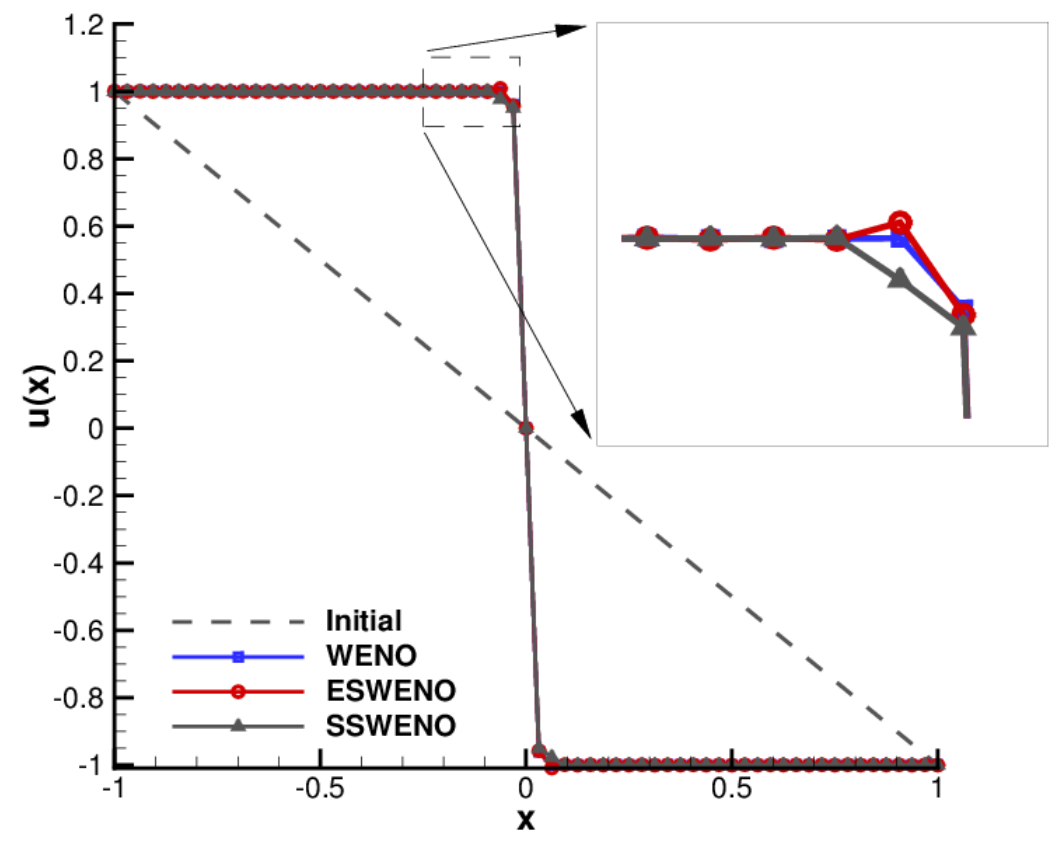

Figure 3. SSWENO, ESWENO, and WENO results for the stationary Burgers shock case are compared for $N=65$ points at $t=2.5$.

for more complex problems. This is demonstrated with a different test problem with time-dependent boundary conditions,

$$
\begin{gathered}
x_{L}=-1, \quad x_{R}=1, \quad \varepsilon=0, \quad t \in[0,5] \\
g_{L}(t)=1+\frac{1}{2} \sin (\pi t), \quad g_{R}(t)=-1-\frac{1}{2} \sin (\pi t), \quad g_{i}(x)=-x .
\end{gathered}
$$

Multiple shocks meet at $x=0$ in this configuration. The problem described by 4.16 was simulated with $N=65$ points and the SSWENO results are compared to the WENO and ESWENO results at $t=2.5$ (see Figure 4). It is clear that SSWENO performs as expected. The overshoots at the shocks disappear, and the one-sided stencil biasing mechanics are well controlled. (Figure 4). This problem also demonstrates how the WENO scheme without correction degenerates at boundaries.

\subsection{Euler and Navier-Stokes Equations}

The end goal of this work is to simulate the Navier-Stokes equations in a stable and efficient manner. Toward this end, the application of entropy stable inviscid and viscous terms are detailed in this section. The developments for the Navier-Stokes 


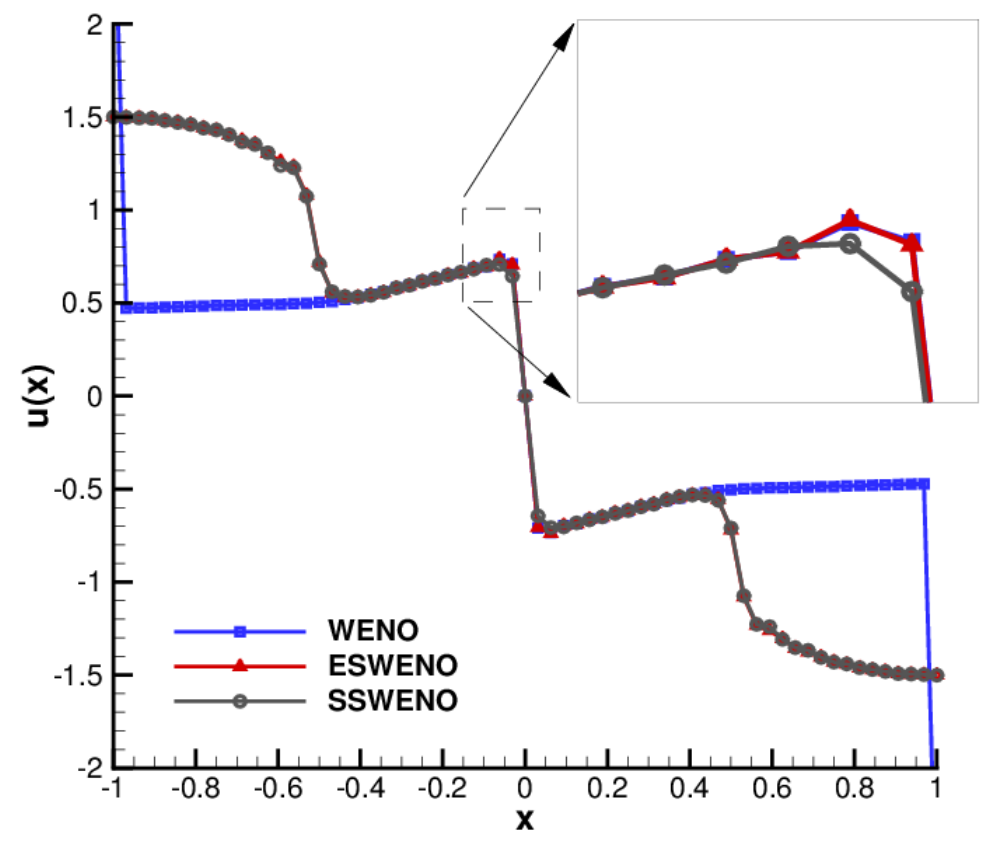

Figure 4. The solutions using SSWENO, ESWENO, and WENO on $N=65$ nodes are plotted for the multiple shock problem at $t=2.5$.

equations in this work are limited to the calorically perfect form,

$$
\begin{gathered}
q_{t}+\left(f^{i}\right)_{x_{i}}=\left(f^{(v) i}\right)_{x_{i}}, \quad x \in \Omega, \quad t \in[0, \infty), \\
B q=g_{b}, \quad x \in \partial \Omega, \quad t \in[0, \infty), \\
q(x, 0)=g_{0}(x), \quad x \in \Omega,
\end{gathered}
$$

where the Cartesian coordinates, $x=\left(x_{1}, x_{2}, x_{3}\right)^{T}$, and time, $t$, are independent variables and index sums are implied. The three dimensional domain is defined by the box

$$
\Omega=\left[x_{1}^{L}, x_{1}^{H}\right] \times\left[x_{2}^{L}, x_{2}^{H}\right] \times\left[x_{3}^{L}, x_{3}^{H}\right]
$$

and $\partial \Omega$ represents the boundary of the domain. The conservative variables are

$$
q=\left(\rho, \rho v_{1}, \rho v_{2}, \rho v_{3}, \rho E\right)^{T},
$$

where $\rho$ denotes density, $v=\left(v_{1}, v_{2}, v_{3}\right)^{T}$ is the velocity vector, and $E$ is the specific total energy. The convective fluxes are

$$
f^{i}=\left(\rho v_{i}, \rho v_{i} v_{1}+\delta_{i 1} p, \rho v_{i} v_{2}+\delta_{i 2} p, \rho v_{i} v_{3}+\delta_{i 3} p, \rho v_{i} H\right)^{T},
$$

where $p$ represents pressure, $H=E+p / \rho$ is the specific total enthalpy, and $\delta_{i j}$ is the Kronecker delta. The viscous flux terms are

$$
f^{(v) i}=\left(0, \tau_{i 1}, \tau_{i 2}, \tau_{i 3}, \tau_{j i} v_{j}-q_{i}\right)^{T},
$$


where the shear stress is

$$
\tau_{i j}=\mu\left(\left(v_{i}\right)_{x_{j}}+\left(v_{j}\right)_{x_{i}}-\delta_{i j} \frac{2}{3}\left(v_{\ell}\right)_{x_{\ell}}\right),
$$

and the heat flux is

$$
q_{i}=-\kappa T_{x_{i}} .
$$

$T$ denotes the static temperature, and $\mu=\mu(T)$, and $\kappa=\kappa(T)$ are the dynamic viscosity and thermal conductivity, respectively. The viscous terms can also be expressed as

$$
f^{(v) i}=c_{i j} q_{x_{j}},
$$

which is a convenient form for entropy analysis. The constitutive relations for a perfect gas are

$$
h=H-\frac{1}{2} v_{j} v_{j}=c_{p} T,
$$

where $c_{p}$ is the constant specific heat, and

$$
p=\rho R T, \quad R=\frac{R_{u}}{M W},
$$

where $R_{u}$ is the universal gas constant and $M W$ is the molecular weight of the gas. The speed of sound for a perfect gas is

$$
c=\sqrt{\gamma R T}, \quad \gamma=\frac{c_{p}}{c_{p}-R} .
$$

In the entropy analysis that follows, the definition of the thermodynamic entropy is the explicit form,

$$
s=\frac{R}{\gamma-1} \log \left(\frac{T}{T_{0}}\right)-R \log \left(\frac{\rho}{\rho_{0}}\right)
$$

\subsubsection{Entropy Analysis}

In the Navier-Stokes equations, entropy stability is not the same as full nonlinear stability. However, entropy stability gives a stronger stability estimate than linear energy stability, and in many ways is easier to apply. In this section, the continuous entropy stability is conducted first to illustrate the entropy characteristics of the governing equations at the continuous level. Then, spatial operators are derived that enable these continuous properties to be mimicked, which is shown through the semi-discrete entropy analysis.

The entropy-entropy flux pair for the Navier-Stokes equations is

$$
S=-\rho s, \quad F^{i}=-\rho v_{i} s,
$$

and the potential-potential flux pair is

$$
\varphi=\rho R, \quad \psi^{i}=\rho v_{i} R
$$


Note again that the mathematical entropy has the opposite sign of the thermodynamic entropy. To avoid confusion, herein entropy refers to the mathematical entropy unless otherwise noted. The entropy variables using the pair in 4.28 are

$$
w=S_{q}^{T}=\left(\frac{h}{T}-s-\frac{v_{j} v_{j}}{2 T}, \frac{v_{1}}{T}, \frac{v_{2}}{T}, \frac{v_{3}}{T},-\frac{1}{T}\right)^{T}
$$

and can be shown to have a one-to-one mapping with the conservative variables as long as $\rho, T>0$. Expressly:

$$
\zeta^{T} S_{q q} \zeta^{T}>0, \quad \forall \zeta \neq 0, \quad \rho, T>0 .
$$

This restriction is what makes the entropy proof fail to be a true measure of nonlinear stability. Another mechanism must be employed to bound $\rho$ and $T$ away from zero to ensure stability. This is not considered in the present work, but interested readers can refer to the work by Zhang and Shu [31]. The full derivation of the entropy variables and symmetrizing matrices are detailed in Appendix B.1.

The entropy equation is found by premultiplying the Navier-Stokes equations with the transpose of the entropy variables,

$$
\begin{aligned}
S_{q} q_{t}+S_{q}\left(f^{i}\right)_{x_{i}}=S_{t}+F_{x_{i}}^{i} & =w^{T}\left(c_{i j} q_{w} w_{x_{j}}\right)_{x_{i}} \\
& =\left(w^{T} c_{i j} q_{w} w_{x_{j}}\right)_{x_{i}}-w_{x_{i}}^{T} c_{i j} q_{w} w_{x_{j}},
\end{aligned}
$$

where the viscous terms satisfy

$$
c_{i j} q_{w}=\hat{c}_{i j}=\hat{c}_{j i}^{T}, \quad \zeta_{x_{i}} \hat{c}_{i j} \zeta_{x_{j}} \geq 0, \quad \forall \zeta
$$

requiring

$$
T>0, \quad \mu(T)>0, \quad \kappa(T)>0 .
$$

This requirement and the symmetric coefficient matrices, $\hat{c}_{i j}$, are derived in Appendix B.2. The total entropy decay rate is found by integrating 4.31 over space,

$$
\frac{d}{d t} \int_{\Omega} S \mathrm{~d} V=\int_{\partial \Omega}\left(w^{T} \hat{c}_{i j} w_{x_{j}}-F^{i}\right) \partial S^{i}-\int_{\Omega} w_{x_{i}}^{T} \hat{c}_{i j} w_{x_{j}} \mathrm{~d} V .
$$

Dutt [32] used the above equation to derive well-posed boundary conditions. His procedure has not gained wide acceptance [33]. In this work, boundary conditions are still imposed based on linear stability theory.

\subsubsection{Discretization Notes}

To facilitate the extension of the entropy stable methods to the three-dimensional equations, we define the three-dimensional nomenclature and examine the general form of the semi-discretization. The semi-discrete form of 4.17 is

$$
\mathbf{u}_{t}+\sum_{i=1}^{3} \mathcal{P}_{x_{i}}^{-1} \Delta_{x_{i}}\left(\overline{\mathbf{f}}^{i}-\overline{\mathbf{f}}^{(v) i}\right)=\sum_{i=1}^{3} \mathcal{P}_{x_{i}}^{-1}\left(\mathbf{g}_{b}^{i}+\mathbf{g}_{I}^{i}\right) .
$$


The solution vector is ordered as

$$
\mathbf{u}=\left(u\left(x_{(1)(1)(1)}\right)^{T}, u\left(x_{(1)(1)(2)}\right)^{T}, \ldots, u\left(x_{\left(N_{1}\right)\left(N_{2}\right)\left(N_{3}\right)}\right)^{T}\right)^{T} .
$$

The roman superscript indices on the flux in 2.43 indicate the direction of the flux, and parenthetic superscripts indicate the type of flux, i.e. $\mathrm{V}$ for viscous, $\mathrm{W}$ for WENO, S for entropy consistent. The multi-dimensional operator nomenclature is defined as follows:

$$
\begin{gathered}
\mathcal{P}_{x_{1}}=\left(\mathcal{P}_{N_{1}} \otimes I_{N_{2}} \otimes I_{N_{3}} \otimes I_{5}\right), \quad \mathcal{P}_{x_{2}}=\left(I_{N_{1}} \otimes \mathcal{P}_{N_{2}} \otimes I_{N_{3}} \otimes I_{5}\right), \\
\mathcal{P}_{x_{3}}=\left(I_{N_{1}} \otimes I_{N_{2}} \otimes \mathcal{P}_{N_{3}} \otimes I_{5}\right), \quad \mathcal{P}_{x_{1} x_{2}}=\left(\mathcal{P}_{N_{1}} \otimes \mathcal{P}_{N_{2}} \otimes I_{N_{3}} \otimes I_{5}\right), \\
\mathcal{P}_{x_{1} x_{3}}=\left(\mathcal{P}_{N_{1}} \otimes I_{N_{2}} \otimes \mathcal{P}_{N_{3}} \otimes I_{5}\right), \quad \mathcal{P}_{x_{2} x_{3}}=\left(I_{N_{1}} \otimes \mathcal{P}_{N_{2}} \otimes \mathcal{P}_{N_{3}} \otimes I_{5}\right), \\
\mathcal{P}=\mathcal{P}_{x_{1} x_{2} x_{3}}=\left(\mathcal{P}_{N_{1}} \otimes \mathcal{P}_{N_{2}} \otimes \mathcal{P}_{N_{3}} \otimes I_{5}\right),
\end{gathered}
$$

where $\otimes$ represents the Kronecker product; $\mathcal{P}_{N}$ represents the one-dimensional norm defined with $N$ solution points; $I_{N}$ is the one-dimensional identity operator defined

with $N$ solution points; and the other operators, $\mathcal{B}, \mathcal{Q}, \Delta, \mathcal{M}, \mathcal{R}$, extend in an identical fashion. Note that transposition and inverse operations applied to these operators are distributive. When applying these operators to the scalar entropy equation in space, a hat will be used to differentiate the scalar operator from the full vector operator. For example,

$$
\hat{\mathcal{P}}=\left(\mathcal{P}_{N_{1}} \otimes \mathcal{P}_{N_{2}} \otimes \mathcal{P}_{N_{3}}\right)
$$

Expressly, 4.35 states that one subscript on an operator means the operator applies in one direction, two subscripts apply in two-directions, and three subscripts or boldface apply in all three directions. This nomenclature is used throughout the following analyses and definitions.

\subsubsection{Entropy Stable Spatial Discretization}

The inviscid terms in the discretization of the Navier-Stokes equations are calculated according to $3.42,3.9$, and 3.10 , where the two-point entropy consistent flux was 
derived by Ismail and Roe [8],

$$
\begin{aligned}
& \left.\bar{f}_{S}^{j}\left(u_{i}, u_{i+1}\right)=\left(\hat{\rho} \hat{v}_{j}, \hat{\rho} \hat{v}_{j} \hat{v}_{1}+\delta_{j 1} \hat{p}, \hat{\rho} \hat{v}_{j} \hat{v}_{2}+\delta_{j 2} \hat{p}, \hat{\rho} \hat{v}_{j} \hat{v}_{3}+\delta_{j 3} \hat{p}, \hat{\rho} \hat{v}_{j} \hat{H}\right)\right)^{T},
\end{aligned}
$$

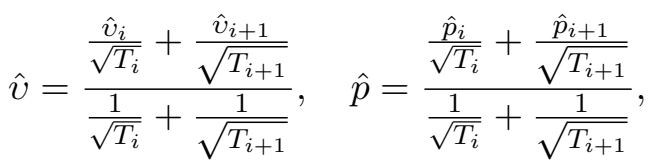

$$
\begin{aligned}
& \hat{h}=R \frac{\log \left(\frac{\sqrt{T_{i}} \rho_{i}}{\sqrt{T_{i+1}} \rho_{i+1}}\right)}{\frac{1}{\sqrt{T_{i}}}+\frac{1}{\sqrt{T_{i+1}}}}\left(\frac{\sqrt{T_{i}} \rho_{i}+\sqrt{T_{i+1}} \rho_{i+1}}{\left(\frac{1}{\sqrt{T_{i}}}+\frac{1}{\sqrt{T_{i+1}}}\right)\left(\sqrt{T_{i}} \rho_{i}-\sqrt{T_{i+1}} \rho_{i+1}\right)}\right. \\
& \left.+\frac{\gamma+1}{\gamma-1} \frac{\log \left(\sqrt{\frac{T_{i+1}}{T_{i}}}\right)}{\log \left(\sqrt{\frac{T_{i}}{T_{i+1}}} \frac{\rho_{i}}{\rho_{i+1}}\right)\left(\frac{1}{\sqrt{T_{i}}}-\frac{1}{\sqrt{T_{i+1}}}\right)}\right), \\
& \hat{H}=\hat{h}+\frac{1}{2} \hat{v}_{\ell} \hat{v}_{\ell}, \quad \hat{\rho}=\frac{\left(\frac{1}{\sqrt{T_{i}}}+\frac{1}{\sqrt{T_{i+1}}}\right)\left(\sqrt{T_{i}} \rho_{i}-\sqrt{T_{i+1}} \rho_{i+1}\right)}{2\left(\log \left(\sqrt{T_{i}} \rho_{i}\right)-\log \left(\sqrt{T_{i+1}} \rho_{i+1}\right)\right)} .
\end{aligned}
$$

This somewhat complicated explicit form is the first entropy consistent flux for the convective terms with low enough computational cost to be implemented in a practical simulation code. Previously, Tadmor [5] derived an entropy consistent flux form that required integration through phase space, but this was deemed too expensive to be practical.

The resulting entropy stable WENO scheme for the Navier-Stokes equations satisfies

$$
\mathbf{w}^{T} \Delta_{x_{i}} \overline{\mathbf{f}}^{(S S W) i} \geq \hat{\mathcal{B}}_{x_{i}} \mathbf{F}^{i}
$$

in each direction. This new version of WENO is more robust than ESWENO for the Navier-Stokes equations and easier to implement. The current major limitation is that it has only been derived for the calorically perfect equations.

The definitions in 4.35 are used to extend the viscous operator in 3.43 to a system of equations in three dimensions. Recall that to demonstrate the entropy decay resulting from the viscous terms, the discrete viscous fluxes must be written in terms of entropy variable gradients,

$$
\begin{gathered}
\left(\hat{c}_{i i} w_{x_{i}}\right)_{x_{i}}=\mathcal{D}_{x_{i} x_{i}}\left(\hat{c}_{i i}\right) \mathbf{w}+\mathcal{T}_{p 2 p p} \\
\mathcal{D}_{x_{i} x_{i}}\left(\hat{c}_{i i}\right) \mathbf{w}=\mathcal{P}_{x_{i}}^{-1}\left(-\mathcal{M}_{x_{i}}\left(\hat{c}_{i i}\right)+\mathcal{B}_{x_{i}}\left[\hat{c}_{i i}\right] \mathcal{D}_{x_{i}}\right) \mathbf{w} \\
\mathcal{M}_{x_{i}}\left(\hat{c}_{i i}\right)=\mathcal{D}_{x_{i}}^{T} \mathcal{P}_{x_{i}}\left[\hat{c}_{i i}\right] \mathcal{D}_{x_{i}}+\mathcal{R}_{x_{i}}\left(\hat{c}_{i i}\right), \quad \mathcal{R}_{x_{i}}\left(\hat{c}_{i i}\right)=\sum_{k=1}^{n_{r}} \mathcal{N}_{k x_{i}}^{T} \widetilde{\left[\hat{c}_{i i}\right]_{k}} \mathcal{N}_{k x_{i}}
\end{gathered}
$$

The definition above only considers the approximation of the diagonal viscous terms, where two derivative operators are applied in the same direction. Cross terms can be incorporated into the same form using the SBP property, $\mathcal{Q}=\mathcal{B}-\mathcal{Q}^{T}$, yielding 
the full viscous flux gradient,

$$
\begin{gathered}
\left(\hat{c}_{i j} w_{x_{i}}\right)_{x_{j}}=\mathcal{D}_{x_{i} x_{j}}\left(\hat{c}_{i j}\right) \mathbf{w}+\mathcal{T}_{e} \\
\mathcal{D}_{x_{i} x_{j}}\left(\hat{c}_{i j}\right) \mathbf{w}=\mathcal{P}_{x_{i}}^{-1}\left(-\mathcal{D}_{x_{i}}^{T} \mathcal{P}_{x_{i}}\left[\hat{c}_{i j}\right] \mathcal{D}_{x_{j}}-\delta_{i j} \mathcal{R}_{x_{j}}\left(\hat{c}_{i j}\right)+\mathcal{B}_{x_{i}}\left[\hat{c}_{i j}\right] \mathcal{D}_{x_{j}}\right) \mathbf{w} \\
\mathcal{R}_{x_{j}}\left(\hat{c}_{i j}\right)=\sum_{k=1}^{n_{r}} \mathcal{N}_{k x_{j}}^{T} \widetilde{\left.\hat{c}_{i j}\right]_{k}} \mathcal{N}_{k x_{j}}
\end{gathered}
$$

We recast the viscous gradient in 4.40 into a flux difference form. The equivalent viscous flux differences are defined by

$$
\Delta_{x_{i}} \overline{\mathbf{f}}^{(v) i}=\sum_{\ell=1}^{3}\left(\mathcal{B}\left[\hat{c}_{i \ell}\right] \mathcal{D}_{x_{\ell}}-\mathcal{D}_{x_{i}}^{T} \mathcal{P}_{x_{i}}\left[\hat{c}_{i \ell}\right] \mathcal{D}_{x_{\ell}}\right) \mathbf{w}-\sum_{\ell=1}^{n_{r}} \mathcal{N}_{\ell x_{i}}^{T} \widetilde{\left.\hat{c}_{i i}\right]_{\ell}} \mathcal{N}_{\ell x_{i}} \mathbf{w}
$$

The derivation of the actual flux form, $\overline{\mathbf{f}}^{(v)}$, is somewhat complicated and long, including two parts. The procedure to calculate the contribution of the diagonal viscous terms to $\overline{\mathbf{f}}^{(v)}$ is detailed in Appendix A.2.1. The procedure to calculate the contribution of the cross terms to $\overline{\mathbf{f}}^{(v)}$ follows from Appendix A.1.1, where the transverse gradients, calculated using SBP finite differences, are simply interpolated to the flux point using the high-order interpolation formulas.

We calculate the contribution of the viscous terms to the semi-discrete entropy decay rate by premultiplying the flux difference in 4.41 by $\mathbf{w}^{T} \mathcal{P}$,

$$
\begin{aligned}
\mathbf{w}^{T} \mathcal{P} \sum_{i=1}^{3} \mathcal{P}_{x_{i}}^{-1} \Delta_{x_{i}} \overline{\mathbf{f}}^{(v) i}= & \sum_{i=1}^{3} \sum_{\ell=1}^{3} \mathbf{w}^{T} \mathcal{P} \mathcal{P}_{x_{i}}^{-1} \mathcal{B}_{x_{i}}\left[\hat{c}_{i \ell}\right] \mathcal{D}_{x_{\ell}} \mathbf{w} \\
& -\sum_{i=1}^{3} \sum_{\ell=1}^{3}\left(\mathcal{D}_{x_{i}} \mathbf{w}\right)^{T} \mathcal{P}\left[\hat{c}_{i \ell}\right]\left(\mathcal{D}_{x_{\ell}} \mathbf{w}\right) \\
& -\sum_{i=1}^{n_{r}} \sum_{\ell=1}^{n_{r}}\left(\mathcal{N}_{\ell x_{i}} \mathbf{w}\right)^{T} \mathcal{P} \mathcal{P}_{x_{i}}^{-1} \widetilde{\left[\hat{c}_{i i}\right]_{\ell}}\left(\mathcal{N}_{\ell x_{i}} \mathbf{w}\right)
\end{aligned}
$$

Reorganizing the gradient terms into a long vector, we show in Appendix B.2 that the latter two terms in 4.42 are always negative semidefinite as long as temperature, viscosity, and thermal conductivity are positive everywhere in the domain.

The multidimensional form of the semi-discrete entropy decay is found by combining the contributions from the entropy stable WENO flux in 3.42 and the entropy 
stable viscous terms in 4.42 . The resulting semi-discrete entropy decay rate is

$$
\begin{aligned}
\mathbf{w} \mathcal{P} \mathbf{u}_{t}=\frac{d}{d t} \mathbf{1}^{T} \hat{\mathcal{P}} \mathbf{S} \leq & \sum_{i=1}^{3} \mathbf{1}^{T} \hat{\mathcal{P}} \hat{\mathcal{P}}_{x_{i}}^{-1} \hat{\mathcal{B}}_{x_{i}} \mathbf{F}^{i}+\sum_{i=1}^{3} \mathbf{w}^{T} \mathcal{P} \mathcal{P}_{x_{i}}^{-1} \mathbf{g}_{b}^{i} \\
& +\sum_{i=1}^{3} \sum_{\ell=1}^{3} \mathbf{w}^{T} \mathcal{P} \mathcal{P}_{x_{i}}^{-1} \mathcal{B}_{x_{i}}\left[\hat{c}_{i \ell}\right] \mathcal{D}_{x_{\ell}} \mathbf{w} \\
& -\sum_{i=1}^{3} \sum_{\ell=1}^{3}\left(\mathcal{D}_{x_{i}} \mathbf{w}\right)^{T} \mathcal{P}\left[\hat{c}_{i \ell}\right]\left(\mathcal{D}_{x_{\ell}} \mathbf{w}\right) \\
& -\sum_{i=1}^{n_{r}} \sum_{\ell=1}^{n_{r}}\left(\mathcal{N}_{\ell x_{i}} \mathbf{w}\right)^{T} \mathcal{P} \mathcal{P}_{x_{i}}^{-1} \widetilde{\left.\hat{c}_{i i}\right]_{\ell}}\left(\mathcal{N}_{\ell x_{i}} \mathbf{w}\right)
\end{aligned}
$$

The last term is design-order small and vanishes as the grid is refined. The other terms are analogous to the terms in 4.33. For high-order finite-difference (HOFD) methods, this is the first known implementation that satisfies this property.

\subsubsection{Energy Stable Boundary Conditions}

All the problems we have studied in this work have been limited to open boundary conditions, which are implemented in the form of Svärd et al. [22] Extensions of these energy stable boundary conditions to walls are detailed in Berg and Nordström [24].

\section{Accuracy Validation and Robustness}

The accuracy and robustness of the algorithms developed herein are tested using two smooth and two discontinuous problems. The smooth problems are the propagation of an isentropic vortex, and the propagation of the viscous shock. Both problems demonstrate the design-order convergence of the new entropy consistent formulation and of the SSWENO scheme. The final two problems (the Sod and Lax shock tube problems) have discontinuous solutions, and test the efficacy of the entropy stable correction terms used in the SSWENO formulation. It is demonstrated that the corrections do not adversely affect the desirable stencil biasing properties of the baseline WENO scheme.

\subsection{Isentropic Vortex}

The isentropic vortex is an exact solution to the Euler equations that has proven to be an excellent test of the accuracy and functionality of the inviscid components of 
a Navier-Stokes solver. It is fully described by

$$
\begin{gathered}
f(x, y, z, t)=1-\left[\left(x-x_{0}-U_{\infty} \cos (\alpha) t\right)^{2}+\left(y-y_{0}-U_{\infty} \sin (\alpha) t\right)^{2}\right], \\
T(x, y, z, t)=\left[1-\epsilon_{v}^{2} M_{\infty}^{2} \frac{\gamma-1}{8 \pi^{2}} \exp (f(x, y, z, t))\right], \quad \rho(x, y, z, t)=T^{\frac{1}{\gamma-1}} \\
u(x, y, z, t)=U_{\infty} \cos (\alpha)-\epsilon_{v} \frac{y-y_{0}-U_{\infty} \sin (\alpha) t}{2 \pi} \exp \left(\frac{f(x, y, z, t)}{2}\right) \\
v(x, y, z, t)=U_{\infty} \sin (\alpha)-\epsilon_{v} \frac{x-x_{0}-U_{\infty} \cos (\alpha) t}{2 \pi} \exp \left(\frac{f(x, y, z, t)}{2}\right) \\
w(x, y, z, t)=0 .
\end{gathered}
$$

In this study the values $U_{\infty}=M_{\infty} c_{\infty}, \epsilon_{v}=5.0, M_{\infty}=0.5$, and $\gamma=1.4$ are used.

\subsubsection{Optimal Accuracy: A Periodic Cartesian Grid Test Case}

Theorem 3.1 proves that design order entropy consistent fluxes can be constructed using a linear combination of two-point entropy fluxes. A critical assumption used in the proof is that the two-point non-dissipative fluxes satisfy Tadmor's integral relation given in equation 3.10. Herein, the non-dissipative Euler fluxes of Ismail and Roe [8] are used, which to our knowledge have not been shown to satisfy Tadmor's integral relation. The first study is designed to detect the presence of order reduction resulting from the use of the Ismail and Roe fluxes in the entropy consistent formulation. A periodic domain is used to eliminate the order reduction associated with $p^{t h}$-order boundary closures in the diagonal norm operators, thus enabling the potential of fully $2 p^{t h}$-order convergence.

The Cartesian grid test case is described by

$$
x \in(-15,15), \quad y \in(-15,15), \quad\left(x_{0}, y_{0}\right)=(0,0), \quad \alpha=0.0, \quad t \geq 0 .
$$

Although periodic boundary conditions were used, note that the actual propagating vortex solution is not periodic. For this domain and the chosen vortex strength, the deviation from periodicity is exponentially small. Discrete error is compared with the exact solution after one full flow through time.

Three grid resolutions are examined using both the fourth- and sixth-order entropy consistent target finite difference schemes and the entropy stable WENO schemes. The error decay, shown in Tables 1 and 2, asymptotes towards the designed rate in each case (i.e., fourth-order and sixth-order respectively). The error for the SSWENO scheme approaches the target central scheme as the grid is refined. The increase in the SSWENO error constant is more pronounced in the sixth-order case, although still within about a factor two of the target entropy consistent FD scheme.

The study provides evidence that the the non-dissipative Euler fluxes of Ismail and Roe [8] do not degrade the formal accuracy of the high-order entropy consistent fluxes.

\subsubsection{Finite Domain Cartesian Grid Test}

Theorems 3.1 and 3.2 provide a recipe to construct design-order entropy consistent fluxes on a finite domain, including points with biased stencils close to each boundary. Having established fully $2 p^{t h}$-order convergence for the interior operator, now 
Table 1. Error convergence is shown for the fourth-order simulation of the periodic isentropic vortex.

\begin{tabular}{c|cc|cc|cc|cc} 
& \multicolumn{4}{|c|}{ Entropy Consistent FD } & \multicolumn{4}{c}{ Entropy Stable WENO FD } \\
\hline Resolution & $L^{2}$ error & $L^{2}$ rate & $L^{\infty}$ error & $L^{\infty}$ rate & $L^{2}$ error & $L^{2}$ rate & $L^{\infty}$ error & $L^{\infty}$ rate \\
\hline $60 \times 60$ & 1.05 & - & 0.38 & - & 0.88 & - & 0.29 & - \\
$120 \times 120$ & $7.60 \mathrm{e}-02$ & 3.79 & $3.19 \mathrm{e}-02$ & 3.58 & $8.85 \mathrm{e}-02$ & 3.32 & $3.38 \mathrm{e}-02$ & 3.12 \\
$240 \times 240$ & $5.01 \mathrm{e}-03$ & 3.92 & $2.11 \mathrm{e}-03$ & 3.92 & $5.97 \mathrm{e}-03$ & 3.89 & $2.81 \mathrm{e}-03$ & 3.59
\end{tabular}

Table 2. Error convergence is shown for the sixth-order simulation of the periodic isentropic vortex.

\begin{tabular}{c|cc|cc|cc|cc} 
& \multicolumn{4}{|c|}{ Entropy Consistent FD } & \multicolumn{4}{c}{ Entropy Stable WENO FD } \\
\hline Resolution & $L^{2}$ error & $L^{2}$ rate & $L^{\infty}$ error & $L^{\infty}$ rate & $L^{2}$ error & $L^{2}$ rate & $L^{\infty}$ error & $L^{\infty}$ rate \\
\hline $60 \times 60$ & 0.31 & - & 0.13 & - & 0.30 & - & 0.11 & - \\
$120 \times 120$ & $6.05 \mathrm{e}-03$ & 5.67 & $2.50 \mathrm{e}-03$ & 5.69 & $1.20 \mathrm{e}-02$ & 4.63 & $5.58 \mathrm{e}-03$ & 4.34 \\
$240 \times 240$ & $9.76 \mathrm{e}-05$ & 5.95 & $3.81 \mathrm{e}-05$ & 6.04 & $2.46 \mathrm{e}-04$ & 5.60 & $1.83 \mathrm{e}-04$ & 4.93
\end{tabular}

a finite-domain Euler vortex test case is used to establish convergence rates for the boundary closures when using a diagonal norm, finite-domain operator.

The finite domain Cartesian grid test is described by

$$
x \in(-5,5), \quad y \in(-5,5), \quad\left(x_{0}, y_{0}\right)=(0,0), \quad \alpha=0.0, \quad t \geq 0 .
$$

Three different grid resolutions are examined, with the vortex located precisely on the boundary when the error measure is evaluated. This measures the effect of the boundary closure, the penalty boundary condition, and the interior scheme. Both the (2-4-2) and (3-6-3) entropy stable WENO finite difference schemes are evaluated. The designed order of accuracy $(p+1)$ is observed for all algorithms as indicated in Tables 3 and 4, in accordance with the classical theorem of Gustafsson [34]. The reduction in order from $2 p$ to $p+1$ relative to the periodic test case results from the order $p$ boundary closures. No explanation is currently available for why the (3-6-3) scheme appears to converge at a super-optimal rate

Table 3. Error convergence is shown for the (2-4-2) simulation of the finite domain isentropic vortex.

\begin{tabular}{c|cc|cc|cc|cc} 
& \multicolumn{4}{|c|}{ Entropy Consistent FD } & \multicolumn{4}{c}{ Entropy Stable WENO FD } \\
\hline Resolution & $L^{2}$ error & $L^{2}$ rate & $L^{\infty}$ error & $L^{\infty}$ rate & $L^{2}$ error & $L^{2}$ rate & $L^{\infty}$ error & $L^{\infty}$ rate \\
\hline $32 \times 32$ & $5.37 \mathrm{e}-02$ & - & $3.17 \mathrm{e}-02$ & - & $5.44 \mathrm{e}-02$ & - & $3.49 \mathrm{e}-02$ & - \\
$64 \times 64$ & $6.14 \mathrm{e}-03$ & 3.13 & $3.51 \mathrm{e}-03$ & 3.18 & $6.26 \mathrm{e}-03$ & 3.12 & $3.51 \mathrm{e}-03$ & 3.31 \\
$128 \times 128$ & $7.43 \mathrm{e}-04$ & 3.05 & $4.63 \mathrm{e}-04$ & 2.92 & $7.46 \mathrm{e}-04$ & 3.07 & $4.72 \mathrm{e}-04$ & 2.90 \\
$256 \times 256$ & $9.01 \mathrm{e}-05$ & 3.04 & $5.95 \mathrm{e}-05$ & 2.96 & $8.39 \mathrm{e}-05$ & 3.15 & $5.52 \mathrm{e}-05$ & 3.10
\end{tabular}

\subsection{Viscous Shock}

The entropy consistent Navier-Stokes formulation developed herein, relies on newly derived narrow stencil viscous terms. In principle, design order convergence should be achieved for all terms including the nonlinear viscous terms in the energy equation. The convection of the viscous shock (an exact solution to the Navier-Stokes equations) is used to test this assertion as well as the functionality of the viscous 
Table 4. Error convergence is shown for the (3-6-3) simulation of the finite domain isentropic vortex.

\begin{tabular}{c|cc|ccc|cc|cc} 
& \multicolumn{4}{|c|}{ Entropy Consistent FD } & \multicolumn{3}{c}{ Entropy Stable WENO FD } \\
\hline Resolution & $L^{2}$ error & $L^{2}$ rate & $L^{\infty}$ error & $L^{\infty}$ rate & $L^{2}$ error & $L^{2}$ rate & $L^{\infty}$ error & $L^{\infty}$ rate \\
\hline $32 \times 32$ & $7.33 \mathrm{e}-02$ & - & $3.65 \mathrm{e}-02$ & - & $7.32 \mathrm{e}-02$ & - & $3.73 \mathrm{e}-02$ & - \\
$64 \times 64$ & $4.30 \mathrm{e}-03$ & 4.09 & $2.10 \mathrm{e}-03$ & 4.12 & $4.95 \mathrm{e}-03$ & 3.89 & $3.03 \mathrm{e}-03$ & 3.62 \\
$128 \times 128$ & $1.94 \mathrm{e}-04$ & 4.47 & $2.08 \mathrm{e}-04$ & 3.34 & $2.53 \mathrm{e}-04$ & 4.29 & $3.80 \mathrm{e}-04$ & 3.00 \\
$256 \times 256$ & $7.72 \mathrm{e}-06$ & 4.65 & $1.79 \mathrm{e}-05$ & 3.54 & $1.09 \mathrm{e}-05$ & 4.54 & $3.09 \mathrm{e}-05$ & 3.62 \\
$512 \times 512$ & $2.83 \mathrm{e}-07$ & 4.77 & $1.01 \mathrm{e}-06$ & 4.15 & $4.19 \mathrm{e}-07$ & 4.70 & $1.71 \mathrm{e}-06$
\end{tabular}

terms in the solver. The nonlinear convection and viscous terms are perfectly balanced in this computation, and thus the shock thickness remains constant, and the shock front is simply advected. The derivation of this problem is available in Fisher [35].

The convergence rate for the viscous shock is evaluated on a Cartesian grid, described by

$$
x \in(-1,1) \times(-0.5,0.5) .
$$

The shock flow is rotated $\alpha=20^{\circ}$ with respect to the $x$-axis. The shock profile is initially located at $x_{s}=-0.5$ with respect to the origin and is simulated until $t=0.25$. The Reynolds number was $R e=10$, and the reference Mach number was $M=2.5$. Table 5 shows the errors for the Cartesian grid. Because the NavierStokes equations are incompletely parabolic in type, the design order convergence rate for the (2-4-2) and (3-6-3) operators should be 3 and 4, respectively. Once again, super-optimal convergence is observed for this test case. No explanation is currently available for why the schemes converge at a super-optimal rate.

All smooth test cases demonstrate the validity of the Theorems 3.1 and 3.2. Furthermore, the SSWENO formulation achieves design order in all cases with only marginal loss in accuracy relative to the baseline target operator.

Table 5. Error convergence is shown for (2-4-2) and (3-6-3) simulations of the viscous shock.

\begin{tabular}{c|cc|cc|cc|cc} 
& \multicolumn{4}{|c|}{ Entropy Stable $(2-4-2)$} & \multicolumn{4}{c}{ Entropy Stable $(3-6-3)$} \\
\hline Resolution & $L^{2}$ error & $L^{2}$ rate & $L^{\infty}$ error & $L^{\infty}$ rate & $L^{2}$ error & $L^{2}$ rate & $L^{\infty}$ error & $L^{\infty}$ rate \\
\hline $48 \times 24$ & $1.74 \mathrm{e}-03$ & - & $5.60 \mathrm{e}-03$ & - & $3.36 \mathrm{e}-04$ & - & $1.57 \mathrm{e}-03$ & - \\
$96 \times 48$ & $1.10 \mathrm{e}-04$ & 3.97 & $4.24 \mathrm{e}-04$ & 3.72 & $7.62 \mathrm{e}-06$ & 5.46 & $6.51 \mathrm{e}-05$ & 4.59 \\
$192 \times 96$ & $7.30 \mathrm{e}-06$ & 3.92 & $3.38 \mathrm{e}-05$ & 3.65 & $2.53 \mathrm{e}-07$ & 4.91 & $3.42 \mathrm{e}-06$
\end{tabular}

\subsection{Shock Tube Problems}

All WENO schemes including those used herein $[10,28,29]$, incorporate elaborate stencil biasing mechanics to achieve nearly monotone solutions in the vicinity of shocks and other discontinuities. It is critically important that the additional dissipation provided by the entropy stabilization terms does not contaminate the desirable attributes of the baseline WENO scheme. To date, all evidence suggests that this it indeed the case (including test cases run on non-Cartesian meshes on curvilinear grids, but not reported here). The shock tube problems shown below are representative of the observed behavior of the SSWENO scheme. 


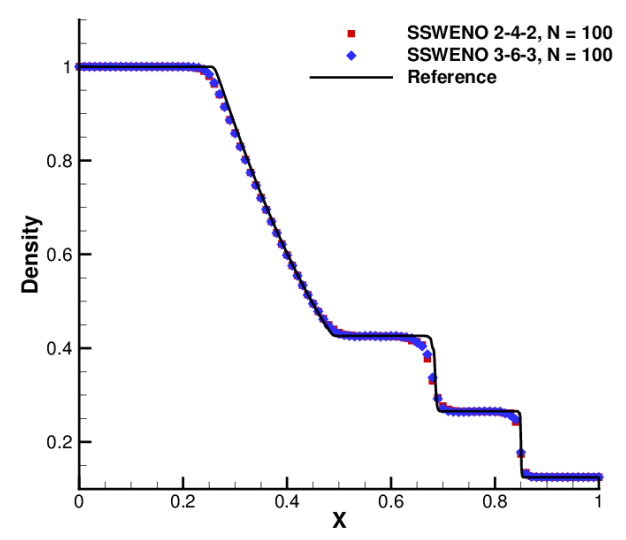

(a) Sod Shock Tube, $t=0.2$

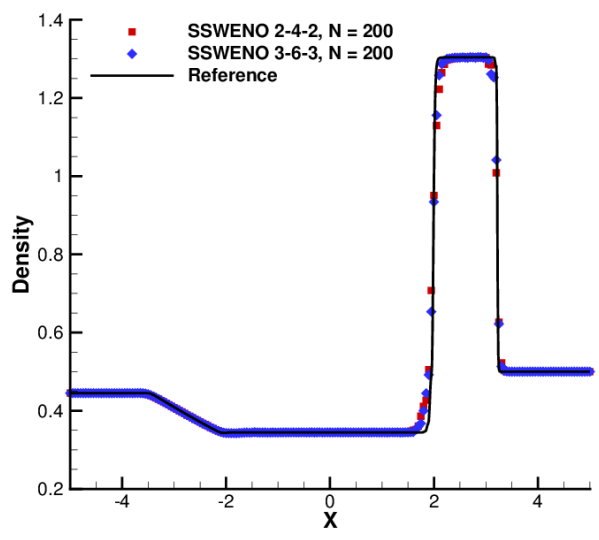

(b) Lax Shock Tube, $t=1.3$

Figure 5. Shock tube solutions are plotted for the entropy stable WENO methods developed in this work and compared to reference solutions.

\subsubsection{Sod Shock Tube}

Sod's shock tube problem evaluates the behavior of a numerical method when a shock, expansion, and contact discontinuity are present. Of particular interest is additional observed smearing in the shock and contact, or oscillations at any of the discontinuities. These would be indications of adverse side-effects of the entropy stabilization term, since the baseline operator performs adequately on this problems.

The domain is

$$
x \in(0,1), \quad y \in(-0.1,0.1), \quad t \geq 0,
$$

and is initialized with

$$
\begin{gathered}
\rho(x, y, z)=\left\{\begin{array}{ll}
1 & x<0.5, \\
1 / 8 & x \geq 0.5,
\end{array} \quad p(x, y, z)= \begin{cases}\gamma & x<0.5, \\
\gamma / 10 & x \geq 0.5,\end{cases} \right. \\
u(x, y, z)=0, \quad v(x, y, z)=0, \quad w(x, y, z)=0,
\end{gathered}
$$

where $\gamma=7 / 5$. The problem is simulated with the (2-4-2) and (3-6-3) entropy stable WENO operators using $N=100$ uniform cells. The solution is plotted for $t=0.2$ in Figure 5(a). The solutions do not exhibit oscillations and the shock smearing is nearly equivalent between the two schemes, with slightly less diffusion observed in the (3-6-3) scheme. All profiles are essentially equivalent to those obtained with the baseline WENO operator.

\subsubsection{Lax Shock Tube}

Lax's shock tube problem is used to show that no entropy problems are observed using the current methodology and that the correct shock location is observed. The reference solution uses $N=800$ points with the (2-4-2) WENO operator. 
The simulated domain is

$$
x \in(-5,5), \quad y \in(-0.5,0.5), \quad t \geq 0,
$$

initialized with

$$
\begin{gathered}
\rho(x, y, z)=\left\{\begin{array}{ll}
0.445 & x<0.0, \\
0.5 & x \geq 0.0,
\end{array} \quad p(x, y, z)= \begin{cases}3.528 \gamma & x<0.0, \\
0.571 \gamma & x \geq 0.0,\end{cases} \right. \\
u(x, y, z)=\left\{\begin{array}{ll}
0.698 & x<0.0, \\
0.0 & x \geq 0.0,
\end{array} \quad v(x, y, z)=0, \quad w(x, y, z)=0,\right.
\end{gathered}
$$

where again $\gamma=7 / 5$. The simulation used $N=200$ uniform cells and the solution is plotted in Figure 5(b) for $t=1.3$. Again the solutions do not exhibit oscillations.

\section{Conclusions}

High-order entropy stable finite difference methods have been derived for conservation laws on finite domains. These methods use formal boundary closures to satisfy a generalized summation-by-parts property. A new method was developed to ensure that dissipative inviscid approximations for shock capturing are guaranteed to be entropy stable. This was shown for WENO but is more generally applicable. A new high-order entropy stable viscous approximation method was also developed using narrow-stencil finite difference operators. These new methods were shown to fix deficiencies observed in linearly stable methods and do not adversely affect shock capturing capability in Burgers equation or the Euler equations. The approach was shown to preserve design-order accuracy of diagonal SBP finite difference operators on simulations of the Euler equations and the Navier-Stokes equations.

\section{References}

1. Shu, C.-W.: High Order Weighted Essentially Nonoscillatory Schemes for Convection Dominated Problems. SIAM Review, vol. 51, 2009, pp. 82-126.

2. Kawai, S.; Shankar, S. K.; and Lele, S. K.: Assessment of localized artificial diffusivity scheme for large-eddy simulation of compressible turbulent flows. Journal of Computational Physics, vol. 229, 2010, pp. 1739-1762.

3. Hill, D. J.; and Pullin, D. I.: Hybrid tuned center-difference-WENO method for large eddy simulations in the presence of strong shocks. Journal of Computational Physics, vol. 194, 2004, pp. 435-450.

4. Lax, P.; and Wendroff, B.: Systems of Conservation Laws. Communications on Pure and Applied Mathematics, vol. 13, 1960, pp. 217-237.

5. Tadmor, E.: The Numerical Viscosity of Entropy Stable Schemes for Systems of Conservation Laws. I. Mathematics of Computation, vol. 49, 1987, pp. 91-103. 
6. Tadmor, E.: Entropy stability theory for difference approximations of nonlinear conservation laws and related time-dependent problems. Acta Numerica, vol. 12, 2003, pp. 451-512.

7. LeFloch, P. G.; Mercier, J. M.; and Rohde, C.: Fully discrete, entropy conservative schemes of arbitrary order. SIAM Journal on Numerical Analysis, vol. 40, 2002, pp. 1968-1992.

8. Ismail, F.; and Roe, P. L.: Affordable, entropy-consistent Euler flux functions II: Entropy production at shocks. Journal of Computational Physics, vol. 228, 2009, pp. 5410-5436.

9. Fjordholm, U. S.; Mishra, S.; and Tadmor, E.: Arbitrarily High-Order Accurate Entropy Stable Essentially Nonoscillatory Schemes for Systems of Conservation Laws. SIAM Journal on Numerical Analysis, vol. 50, 2012, pp. 544-573.

10. Fisher, T. C.; Carpenter, M. H.; Yamaleev, N. K.; and Frankel, S. H.: Boundary closures for fourth-order energy stable weighted essentially non-oscillatory finitedifference schemes. Journal of Computational Physics, vol. 230, 2011, pp. 37273752 .

11. Lax, P. D.: Hyperbolic Systems of Conservation Laws and the Mathematical Theory of Shock Waves. SIAM, Philadelphia, 1973.

12. LeFloch, P. G.: Hyperbolic Systems of Conservation Laws: The Theory of Classical and Nonclassical Shock Waves. Birkhäuser Verlag, Basel, 2002.

13. Harten, A.: On the Symmetric Form of Systems of Conservation Laws with Entropy. Journal of Computational Physics, vol. 49, 1983, pp. 151-164.

14. Hauke, G.; Landaberea, A.; Garmendia, I.; and Canales, J.: On the thermodynamics, stability, and hierarchy of entropy functions in fluid flow. Computer Methods in Applied Mechanics and Engineering, vol. 195, 2006, pp. 4473-4489.

15. Fisher, T. C.; Carpenter, M. H.; Nordström, J.; Yamaleev, N. K.; and Swanson, R. C.: Discretely Conservative Finite-Difference Formulations for Nonlinear Conservation Laws in Split Form: Theory and Boundary Conditions. TM 2011217307, NASA, 2011.

16. Gustafsson, B.: High Order Finite Difference Methods for Time Dependent PDE. Springer-Verlag, Berlin, 2008.

17. Mattsson, K.; and Nordström, J.: Summation by parts operators for finite difference approximations of second derivatives. Journal of Computational Physics, vol. 199, 2004, pp. 503-540.

18. Mattsson, K.; Svärd, M.; and Shoeybi, M.: Stable and accurate schemes for the compressible Navier-Stokes equations. Journal of Computational Physics, vol. 227, 2008, pp. 2293-2316. 
19. Mattsson, K.: Summation by Parts Operators for Finite Difference Approximations of Second-Derivatives with Variable Coefficients. Journal of Scientific Computing, vol. 51, 2012, pp. 650-682.

20. Carpenter, M. H.; Gottlieb, D.; and Abarbanel, S.: Time-Stable Boundary Conditions for Finite-Difference Schemes Solving Hyperbolic Systems: Methodology and Application to High-Order Compact Schemes. Journal of Computational Physics, vol. 111, 1994, pp. 220-236.

21. Svärd, M.; and Nordström, J.: On the order of accuracy for difference approximations of initial-boundary value problems. Journal of Computational Physics, vol. 218, 2006, pp. 333-352.

22. Svärd, M.; Carpenter, M. H.; and Nordström, J.: A stable high-order finite difference scheme for the compressible Navier-Stokes equations, far-field boundary conditions. Journal of Computational Physics, vol. 225, 2007, pp. 1020-1038.

23. Nordström, J.; Gong, J.; van der Weide, E.; and Svärd, M.: A stable and conservative high order multi-block method for the compressible Navier-Stokes equations. Journal of Computational Physics, vol. 228, 2009, pp. 9020-9035.

24. Berg, J.; and Nordström, J.: Stable Robin solid wall boundary conditions for the Navier-Stokes equations. Journal of Computational Physics, vol. 230, 2011, pp. 7519-7532.

25. Randall LeVeque: Numerical Methods for Conservation Laws. Birkhauser, Basel, 1992.

26. Carpenter, M. H.; and Kennedy, C. A.: Fourth-Order 2N-Storage Runge-Kutta Schemes. TM 109112, NASA, 1994.

27. Gottlieb, S.; Shu, C.; and Tadmor, E.: Strong Stability-Preserving High-Order Time Discretization Methods. SIAM Review, vol. 43, no. 1, 2001, pp. 89-112.

28. Yamaleev, N. K.; and Carpenter, M. H.: Third-order Energy stable WENO Scheme. Journal of Computational Physics, vol. 228, 2009, pp. 3025-3047.

29. Yamaleev, N. K.; and Carpenter, M. H.: A systematic methodology for constructing high-order energy stable WENO schemes. Journal of Computational Physics, vol. 228, 2009, pp. 4248-4272.

30. Carpenter, M. H.; Fisher, T. C.; and Yamaleev, N. K.: Boundary Closures for Sixth-Order Energy-Stable Weighted Essentially Non-Oscillatory FiniteDifference Schemes. Advances in Applied Mathematics, Modeling, and Computational Science, R. Melnik and I. S. Kotsireas, eds., Springer, 2013, pp. $117-160$.

31. Zhang, X.; and Shu, C.-W.: On positivity-preserving high order discontinuous Galerkin schemes for compressible Euler equations on rectangular meshes. Journal of Computational Physics, vol. 229, 2010, pp. 8918-8934. 
32. Dutt, P.: Stable Boundary Conditions and Difference Schemes for Navier-Stokes Equations. SIAM Journal on Numerical Analysis, vol. 25, 1988, pp. 245-267.

33. Merriam, M. L.: An Entropy-Based Approach to Nonlinear Stability. TM 101086, NASA, 1989.

34. Gustafsson, B.: The convergence rate for difference approximations to mixed initial boundary value problems. Mathematics of Computation, vol. 29, 1975, pp. 396-406.

35. Fisher, T. C.: High-Order $L^{2}$ Stable Multi-Domain Finite Difference Method for Compressible Flows. Ph.D. Thesis, Purdue University, 2012.

36. Stein, W.; et al.: Sage Mathematics Software (Version 5.0.1). The Sage Development Team, 2012. http://www.sagemath.org. 


\section{Appendix A}

\section{Summation-by-parts operators-(2-4-2)}

The (2-4-2) family of finite difference operators used in this work is specified in this section. This operator set enables the Navier-Stokes equations to be simulated with third-order accuracy. Another important note is that energy and entropy stability proofs require a common diagonal $\mathcal{P}$ norm to be used for all terms in an approximation. The number of special boundary points for this operator is $s=2 p=4$. With this specification, the diagonal $\mathcal{P}$ norm for the (2-4-2) operator is unique,

$$
\mathcal{P}=\operatorname{diag}\left(\frac{17}{48}, \frac{59}{48}, \frac{43}{48}, \frac{49}{48}, 1,1, \cdots, 1,1, \frac{49}{48}, \frac{43}{48}, \frac{59}{48}, \frac{17}{48}\right) \delta x,
$$

where $\mathcal{P}$ is an $(N \times N)$ matrix, and thus has $N$ elements on the diagonal. This form

follows immediately from the accuracy requirement and form of the first derivative operator.

\section{A.1 First Derivative}

Recall that the summation-by-parts operator approximating the first derivative has the form,

$$
\mathcal{D}=\mathcal{P}^{-1} \mathcal{Q}
$$

When using a diagonal norm, $\mathcal{P}$, and $s=2 p$ boundary points, this operator is unique. The matrix elements at the left boundary have the structure,

$$
\mathcal{Q}=\left(\begin{array}{cccccccccc}
-\frac{1}{2} & \frac{59}{96} & -\frac{1}{12} & -\frac{1}{32} & 0 & 0 & 0 & 0 & 0 & \ldots \\
-\frac{59}{96} & 0 & \frac{59}{96} & 0 & 0 & 0 & 0 & 0 & 0 & \ldots \\
\frac{1}{12} & -\frac{59}{96} & 0 & \frac{59}{96} & -\frac{1}{12} & 0 & 0 & 0 & 0 & \ldots \\
\frac{1}{32} & 0 & -\frac{59}{96} & 0 & \frac{2}{3} & -\frac{1}{12} & 0 & 0 & 0 & \ldots \\
0 & 0 & \frac{1}{12} & -\frac{2}{3} & 0 & \frac{2}{3} & -\frac{1}{12} & 0 & 0 & \ldots \\
0 & 0 & 0 & \frac{1}{12} & -\frac{2}{3} & 0 & \frac{2}{3} & -\frac{1}{12} & 0 & \ldots \\
\vdots & \vdots & \vdots & \ddots & \ddots & \ddots & \ddots & \ddots & \ddots & \ddots
\end{array}\right) .
$$

$\mathcal{Q}$ is an $(N \times N)$ matrix and elements corresponding to the right boundary have the property,

$$
q_{(i, j)}=-q_{(N+1-i, N+1-j)}, \quad 1 \leq i, j \leq N .
$$

Each interior row has the form

$$
\begin{gathered}
q_{(i, i-2)}=\frac{1}{12}, \quad q_{(i, i-1)}=-\frac{2}{3}, q_{(i, i+1)}=\frac{2}{3}, \quad q_{(i, i+2)}=-\frac{1}{12}, \\
i=s+1, \ldots, N-s
\end{gathered}
$$




\section{A.1.1 Flux Form}

The first derivative SBP operator above can be recast into the flux form,

$$
\mathcal{Q} \mathbf{f}=\Delta \overline{\mathbf{f}}=\Delta \overline{\mathcal{I}} \mathbf{f}
$$

The interpolation matrix, $\overline{\mathcal{I}}$, is an $(N+1 \times N)$ matrix where each row sums to 1 . The matrix elements near the left boundary are

$$
\overline{\mathcal{I}}=\left(\begin{array}{cccccccccc}
1 & 0 & 0 & 0 & 0 & 0 & 0 & 0 & 0 & \\
\frac{1}{2} & \frac{59}{96} & -\frac{1}{12} & -\frac{1}{32} & 0 & 0 & 0 & 0 & 0 & \ldots \\
-\frac{11}{96} & \frac{59}{96} & \frac{17}{32} & -\frac{1}{32} & 0 & 0 & 0 & 0 & 0 & \ldots \\
-\frac{1}{32} & 0 & \frac{17}{32} & \frac{7}{12} & -\frac{1}{12} & 0 & 0 & 0 & 0 & \ldots \\
0 & 0 & -\frac{1}{12} & \frac{7}{12} & \frac{7}{12} & -\frac{1}{12} & 0 & 0 & 0 & \ldots \\
0 & 0 & 0 & -\frac{1}{12} & \frac{7}{12} & \frac{7}{12} & -\frac{1}{12} & 0 & 0 & \ldots \\
0 & 0 & 0 & 0 & -\frac{1}{12} & \frac{7}{12} & \frac{7}{12} & -\frac{1}{12} & 0 & \ldots \\
\vdots & \vdots & \vdots & \vdots & \vdots & \vdots & \vdots & \vdots & \vdots & \ddots
\end{array}\right) .
$$

The flux consistency condition is imposed in the first row of $\overline{\mathcal{I}}$. The right boundary terms satisfy the matrix property,

$$
\bar{h}_{(i, j)}=\bar{h}_{(N-i, N+1-j)}, \quad 0 \leq i \leq N, \quad 1 \leq j \leq N
$$

where $\bar{h}_{(i, j)}$ is the element in row $i$ and column $j$ in $\overline{\mathcal{I}}$. Note that for the interpolation matrix the first row is indexed at 0 to be consistent with the flux point nomenclature used throughout this document. The interior elements have the formula,

$$
\begin{gathered}
\bar{h}_{(i, i-1)}=-\frac{1}{12}, \quad \bar{h}_{(i, i)}=\frac{7}{12}, \quad \bar{h}_{(i, i+1)}=\frac{7}{12}, \quad \bar{h}_{(i, i+2)}=-\frac{1}{12}, \\
i=s, \ldots, n-s .
\end{gathered}
$$

\section{A.2 Variable Coefficient Second Derivative}

To find the matrices specified in $2.37, \mathcal{N}_{k}$ and $[\tilde{\vartheta}]_{k}$, only $\vartheta(x)=1$ must be considered. Then, for variable coefficients and nonlinear problems, the structure of $[\tilde{\vartheta}]_{k}$ ensures the accuracy and stability of the full operator. The structure of $\mathcal{M}(1)$ is a pentadiagonal matrix with $(s \times s)$ block matrices superimposed on the diagonal at the two boundaries. The first constraint is to require $\mathcal{M}(\mathbf{1})$ to satisfy the optimal accuracy,

$$
\phi_{x x}(\mathbf{x})=\mathcal{P}^{-1}(-\mathcal{M}(1)+\mathcal{B D}) \phi+\mathcal{T}_{2-4-2} .
$$

At fourth-order, this leaves one free parameter in the matrix $\mathcal{M}(1)$. The remainder is constructed using the matrices:

$$
\mathcal{N}_{1}=\left(\begin{array}{cccccccccc}
-1 & 3 & -3 & 1 & 0 & 0 & 0 & 0 & \ldots & 0 \\
0 & -1 & 3 & -3 & 1 & 0 & 0 & 0 & \ldots & 0 \\
0 & 0 & -1 & 3 & -3 & 1 & 0 & 0 & \ldots & 0 \\
\vdots & \ldots & \ddots & \ddots & \ddots & \ddots & \ddots & \ddots & \ldots & \vdots \\
0 & \ldots & 0 & 0 & -1 & 3 & -3 & 1 & 0 & 0 \\
0 & \ldots & 0 & 0 & 0 & -1 & 3 & -3 & 1 & 0 \\
0 & \ldots & 0 & 0 & 0 & 0 & -1 & 3 & -3 & 1
\end{array}\right)
$$


which is a $(N-3 \times N)$ matrix,

$$
\mathcal{N}_{2}=\left(\begin{array}{ccccccccccc}
1 & -4 & 6 & -4 & 1 & 0 & 0 & 0 & 0 & \ldots & 0 \\
0 & 1 & -4 & 6 & -4 & 1 & 0 & 0 & 0 & \ldots & 0 \\
0 & 0 & 1 & -4 & 6 & -4 & 1 & 0 & 0 & \ldots & 0 \\
\vdots & \ldots & \ddots & \ddots & \ddots & \ddots & \ddots & \ddots & \ddots & \ldots & \vdots \\
0 & \ldots & 0 & 0 & 1 & -4 & 6 & -4 & 1 & 0 & 0 \\
0 & \ldots & 0 & 0 & 0 & 1 & -4 & 6 & -4 & 1 & 0 \\
0 & \ldots & 0 & 0 & 0 & 0 & 1 & -4 & 6 & -4 & 1
\end{array}\right)
$$

which is a $(N-4 \times N)$ matrix, and

$$
\mathcal{N}_{3}=\left(\begin{array}{cccccccccccc}
-1 & 5 & -10 & 10 & -5 & 1 & 0 & 0 & 0 & 0 & \ldots & 0 \\
0 & -1 & 5 & -10 & 10 & -5 & 1 & 0 & 0 & 0 & \ldots & 0 \\
0 & 0 & -1 & 5 & -10 & 10 & -5 & 1 & 0 & 0 & \ldots & 0 \\
\vdots & \ldots & \ddots & \ddots & \ddots & \ddots & \ddots & \ddots & \ddots & \ddots & \ldots & \vdots \\
0 & \ldots & 0 & 0 & -1 & 5 & -10 & 10 & -5 & 1 & 0 & 0 \\
0 & \ldots & 0 & 0 & 0 & -1 & 5 & -10 & 10 & -5 & 1 & 0 \\
0 & \ldots & 0 & 0 & 0 & 0 & -1 & 5 & -10 & 10 & -5 & 1
\end{array}\right)
$$

which is a $(N-5 \times N)$ matrix. If only these matrices are used to construct the remainder, a proof that the remainder is positive semi-definite will not be possible. We find that an additional $(N \times N)$ matrix, $\mathcal{N}_{4}$ is required with only the first and last rows containing nonzero terms,

$$
\begin{gathered}
\tilde{n}_{(1,1)}^{(4)}=-a_{1}+a_{2}-a_{3}, \quad \tilde{n}_{(1,2)}^{(4)}=3 a_{1}-4 a_{2}+5 a_{3}, \\
\tilde{n}_{(1,3)}^{(4)}=-3 a_{1}+6 a_{2}-10 a_{3}, \quad \tilde{n}_{(1,4)}^{(4)}=a_{1}-4 a_{2}+10 a_{3}, \\
\tilde{n}_{(1,5)}^{(4)}=a_{2}-5 a_{3}, \quad \tilde{n}_{(1,6)}^{(4)}=a_{3}, \quad \tilde{n}_{(N, j)}=\tilde{n}_{(1, N+1-j)}, \quad j=1,2, \ldots, N .
\end{gathered}
$$

This introduces nonlinearity into the derivation of the operator, which is addressed differently in our methodology below. The form of the variable coefficient matrices is

$$
\begin{gathered}
\left([\tilde{\vartheta}]_{1}\right)_{(i, i)}=-c_{i}^{(1)} \frac{1}{2}\left(\vartheta_{i+1}+\vartheta_{i+2}\right), \quad i=1,2, \ldots, N-3, \\
\left([\tilde{\vartheta}]_{2}\right)_{(i, i)}=-c_{i}^{(2)} \vartheta_{i+2}, \quad i=1,2, \ldots, N-4, \\
\left([\tilde{\vartheta}]_{3}\right)_{(i, i)}=-c_{i}^{(3)} \frac{1}{2}\left(\vartheta_{i+2}+\vartheta_{i+3}\right), \quad i=1,2, \ldots, N-5, \\
\left([\tilde{\vartheta}]_{4}\right)_{(i, i)}=\vartheta_{i}, \quad i=1,2, \ldots, N,
\end{gathered}
$$

and for the operator to be provably stable, all coefficients, $c_{i}^{(j)} \leq 0$. For $\vartheta(x)=1$, only these coefficients are left in $[\tilde{\vartheta}]_{k}$. The coefficients, $c_{i}^{(j)}$ can be solved for linearly as nonlinear functions of the free parameter left in $\mathcal{M}(1), a_{1}, a_{2}$, and $a_{3}$, such that the remainder term recovers the narrow form of $\mathcal{M}(1)$ with the accuracy constraints 
satisfied. The resulting coefficients, organized into vectors are:

$$
c^{(1)}=\left(\begin{array}{c}
a_{1}^{2}-a_{1} a_{2}+a_{1} a_{3}+\frac{1}{3} m_{(2,4)}-\frac{181507}{1719312} \\
a_{1} a_{2}-2 a_{1} a_{3}-\frac{293}{4214} \\
a_{1} a_{3}-\frac{185}{3528} \\
-\frac{1}{18} \\
-\frac{1}{18} \\
-\frac{1}{18} \\
-\frac{1}{18} \\
-\frac{1}{18} \\
\vdots \\
-\frac{1}{18} \\
-\frac{1}{18} \\
-\frac{1}{18} \\
-\frac{1}{18} \\
-\frac{1}{18} \\
a_{1} a_{3}-\frac{185}{3528} \\
a_{1} a_{2}-2 a_{1} a_{3}-\frac{293}{4214} \\
a_{1}^{2}-a_{1} a_{2}+a_{1} a_{3}+\frac{1}{3} m_{(2,4)}-\frac{181507}{1719312}
\end{array}\right),
$$

and

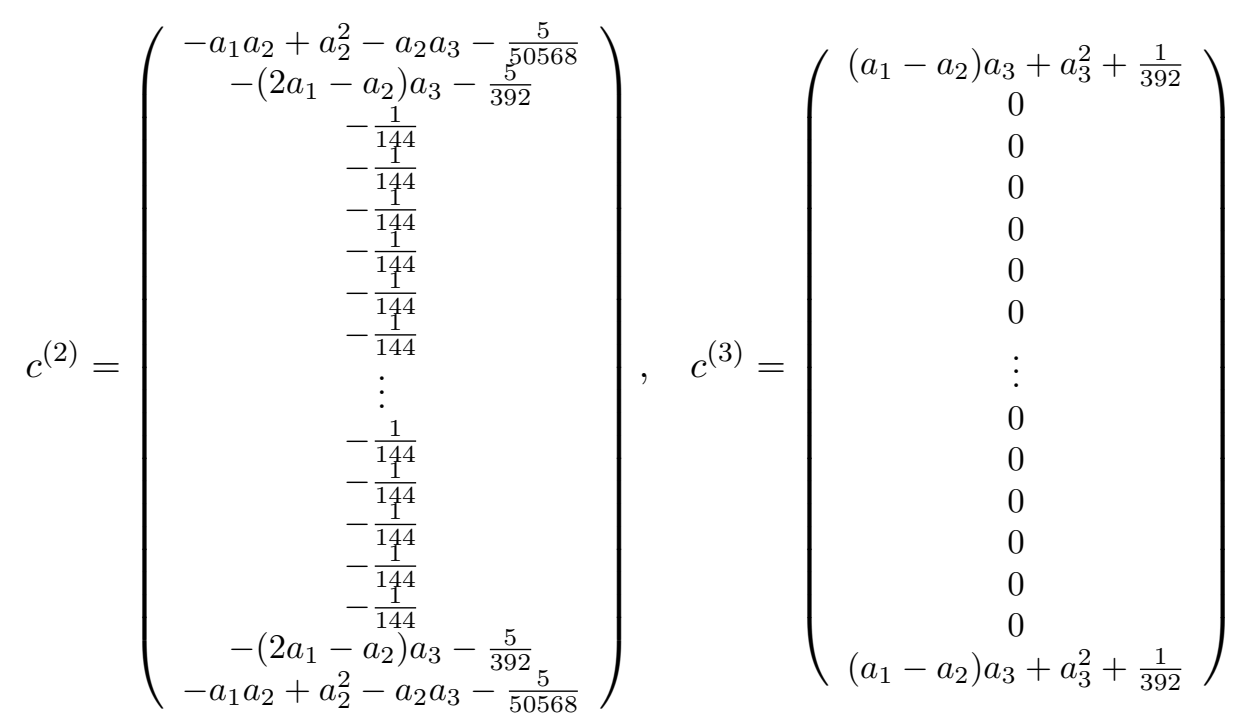

To eliminate the remaining nonlinear terms such that $c_{i}^{(j)} \leq 0$, a nonlinear optimization problem is solved, where the functional is

$$
r=\sum_{i} \sum_{j} \sqrt{\left(c_{i}^{(j)}\right)^{2}+\delta}+c_{i}^{(j)},
$$

which is very small as long as all $c_{i}^{(j)}$ are negative or zero. The functional is minimized using a conjugate gradient method in the open-source computational mathe- 
matics package SAGE [36],

$$
\begin{gathered}
m_{(2,4)}=\frac{16815244}{410099621}, \quad a_{1}=-\frac{88998127}{304807400}, \\
a_{2}=\frac{6823462}{373821039}, \quad a_{3}=-\frac{20751280}{551691433} .
\end{gathered}
$$

The constant coefficient narrow stencil matrix has the form,

$$
\mathcal{M}(\mathbf{1})=\left(\begin{array}{cccccccc}
-\frac{1}{3} m_{(2,4)}+\frac{9}{8} & m_{(2,4)}-\frac{59}{48} & -m_{(2,4)}+\frac{1}{12} & \frac{1}{3} m_{(2,4)}+\frac{1}{48} & 0 & 0 & 0 & \ldots \\
m_{(2,4)}-\frac{59}{48} & -3 m_{(2,4)}+\frac{59}{44} & 3 m_{(2,4)}-\frac{59}{48} & -m_{(2,4)} & 0 & 0 & 0 & \ldots \\
-m_{(2,4)}+\frac{1}{12} & 3 m_{(2,4)}-\frac{59}{48} & -3 m_{(2,4)}+\frac{55}{24} & m_{(2,4)}-\frac{59}{48} & \frac{1}{12} & 0 & 0 & \ldots \\
\frac{1}{3} m_{(2,4)}+\frac{1}{48} & -m_{(2,4)} & m_{(2,4)}-\frac{59}{48} & -\frac{1}{3} m_{(2,4)}+\frac{59}{24} & -\frac{4}{3} & \frac{1}{12} & 0 & \ldots \\
0 & 0 & \frac{1}{12} & -\frac{4}{3} & \frac{5}{2} & -\frac{4}{3} & \frac{1}{12} & \ldots \\
\vdots & \vdots & \vdots & \vdots & \vdots & \vdots & \vdots & \ddots
\end{array}\right),
$$

While thus far only the constant coefficient case has been considered, the variable coefficient viscous gradient operator has been fully specified. It is noted, however, that the number of special boundary points has increased from $s=4$ to $s_{\vartheta}=6$ because the form used here is not optimal for the boundaries. This was deemed appropriate for the time being but will be revisited in the future. The accuracy of the variable coefficient gradient is guaranteed by using only undivided third derivative approximations and above. The lowest-order remainder term is $\mathcal{O}\left(\delta x^{4}\right)$. Higherorder methods can follow in a similar way.

\section{A.2.1 Flux Form}

The operators above are inconvenient for implementation. Instead, the viscous flux gradient is calculated using the flux form,

$$
\mathcal{D}_{2}(\boldsymbol{\vartheta}) \mathbf{v}=\mathcal{P}^{-1} \Delta \overline{\mathbf{f}}^{(v)},
$$

where $\overline{\mathbf{f}}^{(v)}$ is consistent with the viscous flux to design order. To calculate the fluxes, a coefficient array is needed for each flux point, and a three-dimensional array is required to describe the full operator. To reduce confusion, a compressed sparse row structure is used below to show coefficients. The pointer for the beginning of each row is denoted by $z$. The two column pointers corresponding to the coefficient and the variable are denoted by $o^{(1)}$ and $o^{(2)}$, respectively. Note that there are two because of the three-dimensional coefficient array. The coefficients are denoted by $b$. The form of the viscous flux is

$$
\bar{f}_{i}^{(v)}=\sum_{j=1}^{N} \sum_{k=1}^{N} C_{(i, j, k)} \vartheta_{j} v_{k}=\sum_{\ell=z_{i}}^{z_{i+1}-1} b_{\ell} \vartheta_{o_{\ell}^{(1)}} v_{o_{\ell}^{(2)}}, \quad 0 \leq i \leq N .
$$

Note that the structure of the coefficients is such that

$$
C_{(i, j, k)}=C_{(N+1-i, N+1-j, N+1-k)}, \quad 0 \leq i \leq N, \quad 1 \leq j, k \leq N .
$$


The left boundary and first interior points are described by

$$
z=(1,5,27,49,75,100,130,144),
$$

and

$$
\begin{array}{llll}
k & o_{k}^{(1)} & o_{k}^{(2)} & b_{k} \\
1 & 1 & 1 & -1.83333333333333333 \\
2 & 1 & 2 & 3.00000000000000000 \\
3 & 1 & 3 & -1.5000000000000000 \\
4 & 1 & 4 & 0.333333333333333333 \\
5 & 1 & 1 & -0.761632318953671114 \\
6 & 2 & 1 & -0.318772132024516656 \\
7 & 3 & 1 & -0.0290918865391788973 \\
8 & 4 & 1 & -0.00183605333033583864 \\
9 & 1 & 2 & 1.01739604757603651 \\
10 & 2 & 2 & 0.0344413960735499694 \\
11 & 3 & 2 & 0.131929292173814632 \\
12 & 4 & 2 & 0.00439710338637307075 \\
13 & 1 & 3 & -0.228084367243370364 \\
14 & 2 & 3 & 0.272850270593116697 \\
15 & 3 & 3 & -0.0971159779636451267 \\
16 & 4 & 3 & 0.0100195687374579401 \\
17 & 1 & 4 & -0.0537999941893968519 \\
18 & 2 & 4 & 0.0114804653578499898 \\
19 & 3 & 4 & -0.000975620426830106209 \\
20 & 4 & 4 & 0.00879420677274614150 \\
21 & 1 & 5 & 0.0304304945731143092 \\
22 & 3 & 5 & -0.00562522792143511602 \\
23 & 4 & 5 & -0.0248052666516791932 \\
24 & 1 & 6 & -0.00430986176271249361 \\
25 & 3 & 6 & 0.000879420677274614150 \\
26 & 4 & 6 & 0.00343044108543787946 \\
27 & 1 & 1 & 0.255763728622365400 \\
28 & 2 & 1 & -0.284330735950966687 \\
29 & 3 & 1 & 0.102837405634635735 \\
30 & 4 & 1 & 0.00256105005603723211 \\
31 & 1 & 2 & -0.451324881722072318 \\
32 & 2 & 2 & -0.0688827921470999388 \\
33 & 3 & 2 & -0.582638326788311979 \\
34 & 4 & 2 & -0.0443150110953974708 \\
35 & 1 & 3 & 0.213167159097448528 \\
36 & 2 & 3 & 0.376174458813766606 \\
37 & 3 & 3 & 0.337533229266219984 \\
38 & 4 & 3 & 0.136952831242113255 \\
39 & 1 & 4 & 0.0264355268164343218 \\
40 & 2 & 4 & -0.0229609307156999796 \\
41 & 3 & 4 & 0.126731216584854573 \\
42 & 4 & 4 & -0.123703927714327261 \\
43 & 1 & 5 & -0.0513083138154186242 \\
44 & 3 & 5 & 0.0190541580117001441 \\
45 & 4 & 5 & 0.0322541558037184802 \\
46 & 1 & 6 & 0.00726678100124269184 \\
47 & 3 & 6 & -0.00351768270909845660 \\
48 & 4 & 6 & -0.00374909829214423523 \\
49 & 1 & 1 & 0.0276793613789950362 \\
50 & 2 & 1 & -0.0114804653578499898 \\
51 & 3 & 1 & 0.00572142767099060808 \\
52 & 4 & 1 & 0.0125806187934951722 \\
53 & 1 & 2 & -0.0100733553812534256 \\
54 & 2 & 2 & 0.0344413960735499694 \\
55 & 3 & 2 & -0.147989119558446868 \\
56 & 4 & 2 & 0.0826182514092578440
\end{array}
$$




\begin{tabular}{|c|c|c|c|}
\hline$k$ & $o_{k}^{(1)}$ & $o_{k}^{(2)}$ & $b_{k}$ \\
\hline 57 & $1^{n}$ & $3^{n}$ & -0.0252103078326823143 \\
\hline 58 & 2 & 3 & -0.0344413960735499694 \\
\hline 59 & 3 & 3 & -0.392512731414250729 \\
\hline 60 & 4 & 3 & -0.609893549870353691 \\
\hline 61 & 5 & 3 & -0.0427725206856041496 \\
\hline 62 & 1 & 4 & -0.0000975580043828657740 \\
\hline 63 & 2 & 4 & 0.0114804653578499898 \\
\hline 64 & 3 & 4 & 0.680133634883527315 \\
\hline 65 & 4 & 4 & 0.333164953220562285 \\
\hline 66 & 5 & 4 & 0.169984228723479116 \\
\hline 67 & 1 & 5 & 0.00897265413685119573 \\
\hline 68 & 3 & 5 & -0.150629735645468011 \\
\hline 69 & 4 & 5 & 0.186641310232095931 \\
\hline 70 & 5 & 5 & -0.128317562056812449 \\
\hline 71 & 1 & 6 & -0.00127079429752762628 \\
\hline 72 & 3 & 6 & 0.00527652406364768490 \\
\hline 73 & 4 & 6 & -0.00511158378505754159 \\
\hline 74 & 5 & 6 & 0.00110585401893748296 \\
\hline 75 & 1 & 1 & -0.0261206328104018156 \\
\hline 76 & 3 & 1 & 0.00474580724416050187 \\
\hline 77 & 4 & 1 & 0.0213748255662413137 \\
\hline 78 & 1 & 2 & 0.0701621656245777480 \\
\hline 79 & 3 & 2 & -0.0202822825467621893 \\
\hline 80 & 4 & 2 & -0.0498798830778155587 \\
\hline 81 & 1 & 3 & -0.0517433926534995019 \\
\hline 82 & 3 & 3 & 0.160889686884422013 \\
\hline 83 & 4 & 3 & -0.153024668935464144 \\
\hline 84 & 5 & 3 & 0.127211708037874966 \\
\hline 85 & 1 & 4 & -0.00453648768294982641 \\
\hline 86 & 3 & 4 & -0.200100104480964590 \\
\hline 87 & 4 & 4 & -0.330394950389127353 \\
\hline 88 & 5 & 4 & -0.673301790780291564 \\
\hline 89 & 6 & 4 & -0.0416666666666666667 \\
\hline 90 & 1 & 5 & 0.0142576548800962444 \\
\hline 91 & 3 & 5 & 0.0582645756082427202 \\
\hline 92 & 4 & 5 & 0.629175978731369471 \\
\hline 93 & 5 & 5 & 0.381635124113624898 \\
\hline 94 & 6 & 5 & 0.166666666666666667 \\
\hline 95 & 1 & 6 & -0.00201930735782284852 \\
\hline 96 & 3 & 6 & -0.00351768270909845660 \\
\hline 97 & 4 & 6 & -0.117251301895203729 \\
\hline 98 & 5 & 6 & 0.164454958628791701 \\
\hline 99 & 6 & 6 & -0.125000000000000000 \\
\hline 100 & 1 & 1 & 0.00430986176271249361 \\
\hline 101 & 3 & 1 & -0.000879420677274614150 \\
\hline 102 & 4 & 1 & -0.00343044108543787946 \\
\hline 103 & 1 & 2 & -0.0115766427639551854 \\
\hline 104 & 3 & 2 & 0.00439710338637307075 \\
\hline 105 & 4 & 2 & 0.00717953937758211469 \\
\hline 106 & 1 & 3 & 0.00853757529877031811 \\
\hline 107 & 3 & 3 & -0.00879420677274614150 \\
\hline 108 & 4 & 3 & 0.00136248549291330635 \\
\hline 109 & 5 & 3 & -0.00110585401893748296 \\
\hline 110 & 1 & 4 & 0.000748513060295222244 \\
\hline 111 & 3 & 4 & 0.00879420677274614150 \\
\hline 112 & 4 & 4 & 0.112139718110146187 \\
\hline
\end{tabular}




$\begin{array}{llll}k & o_{k}^{(1)} & o_{k}^{(2)} & b_{k} \\ 113 & 5 & 4 & -0.163349104609854218 \\ 114 & 6 & 4 & 0.12500000000000000 \\ 115 & 1 & 5 & -0.00235248977464312509 \\ 116 & 3 & 5 & -0.00439710338637307075 \\ 117 & 4 & 5 & -0.156599511448838022 \\ 118 & 5 & 5 & -0.378317562056812449 \\ 119 & 6 & 5 & -0.666666666666666667 \\ 120 & 7 & 5 & -0.0416666666666666667 \\ 121 & 1 & 6 & 0.000333182416820276572 \\ 122 & 3 & 6 & 0.000879420677274614150 \\ 123 & 4 & 6 & 0.0393482095536342930 \\ 124 & 5 & 6 & 0.667772520685604150 \\ 125 & 6 & 6 & 0.375000000000000000 \\ 126 & 7 & 6 & 0.166666666666666667 \\ 127 & 5 & 7 & -0.125000000000000000 \\ 128 & 6 & 7 & 0.166666666666666667 \\ 129 & 7 & 7 & -0.125000000000000000 \\ 130 & 5 & 5 & 0.125000000000000000 \\ 131 & 6 & 5 & -0.166666666666666667 \\ 132 & 7 & 5 & 0.125000000000000000 \\ 133 & 5 & 6 & -0.166666666666666667 \\ 134 & 6 & 6 & -0.375000000000000000 \\ 135 & 7 & 6 & -0.666666666666666667 \\ 136 & 8 & 6 & -0.0416666666666666667 \\ 137 & 5 & 7 & 0.0416666666666666667 \\ 138 & 6 & 7 & 0.666666666666666667 \\ 139 & 7 & 7 & 0.375000000000000000 \\ 140 & 8 & 7 & 0.166666666666666667 \\ 141 & 6 & 8 & -0.125000000000000000 \\ 142 & 7 & 8 & 0.166666666666666667 \\ 143 & 8 & 8 & -0.125000000000000000\end{array}$




\section{Appendix B}

\section{Navier-Stokes Equations-Supplemental Details}

\section{B.1 Derivation of Entropy Variables}

Recall that the entropy variables are defined as $w^{T}=S_{q}$. While this can be calculated directly for the calorically perfect equations, in general it is more convenient to use the primitive variables to aid in the derivation. To accomplish this, the standard differential relations for pressure, enthalpy, internal energy, and entropy are used,

$$
\begin{gathered}
d p=\rho R d T+R T d \rho, \\
d h=\frac{\gamma R}{\gamma-1} d T, \quad d e=\frac{R}{\gamma-1} d T \\
d s=\frac{d h}{T}-\frac{d p}{\rho T}=\frac{R d T}{(\gamma-1) T}-R \frac{d \rho}{\rho} .
\end{gathered}
$$

A set of primitive variables,

$$
v=\left(\rho, v_{1}, v_{2}, v_{3}, T\right)^{T},
$$

is selected for the derivation. The expansion $S_{q}=S_{v} v_{q}$ is used, where

$$
S_{v}=\left(R-s, 0,0,0,-\frac{\rho R}{(\gamma-1) T}\right)
$$

is combined with

$$
v_{q}=\left(\begin{array}{rrrrr}
1 & 0 & 0 & 0 & 0 \\
-\frac{v_{1}}{\rho} & \frac{1}{\rho} & 0 & 0 & 0 \\
-\frac{v_{2}}{\rho} & 0 & \frac{1}{\rho} & 0 & 0 \\
-\frac{v_{3}}{\rho} & 0 & 0 & \frac{1}{\rho} & 0 \\
\frac{(\gamma-1)\left(2 R T+v_{1}^{2}+v_{2}^{2}+v_{3}^{2}-2 h\right)}{2 R \rho} & -\frac{(\gamma-1) v_{1}}{R \rho} & -\frac{(\gamma-1) v_{2}}{R \rho} & -\frac{(\gamma-1) v_{3}}{R \rho} & \frac{\gamma-1}{R \rho}
\end{array}\right)
$$

yielding

$$
S_{q}=w^{T}=\left(\frac{h}{T}-s-\frac{v_{k} v_{k}}{2 T}, \frac{v_{1}}{T}, \frac{v_{2}}{T}, \frac{v_{3}}{T},-\frac{1}{T}\right) .
$$

A similar procedure is followed to find the matrix $w_{q}=S_{q q}=w_{v} v_{q}$, where

$$
w_{v}=\left(\begin{array}{rrrrr}
\frac{R}{\rho} & -\frac{u_{1}}{T} & -\frac{u_{2}}{T} & -\frac{u_{3}}{T} & \frac{R}{T}+\frac{u_{1}^{2}}{2 T^{2}}+\frac{u_{2}^{2}}{2 T^{2}}+\frac{u_{3}^{2}}{2 T^{2}}-\frac{h}{T^{2}} \\
0 & \frac{1}{T} & 0 & 0 & -\frac{u_{1}}{T^{2}} \\
0 & 0 & \frac{1}{T} & 0 & -\frac{u_{2}}{T^{2}} \\
0 & 0 & 0 & \frac{1}{T} & -\frac{u_{3}}{T^{2}} \\
0 & 0 & 0 & 0 & \frac{1}{T^{2}}
\end{array}\right) .
$$


To illustrate why $\rho, T>0$ are required for the convexity of the entropy function to hold, the symmetric matrix $w_{q}$ is diagonalized by

$$
D=q_{v}^{T} w_{q} q_{v}=q_{v}^{T} w_{v}=\operatorname{diag}\left(\frac{R}{\rho}, \frac{\rho}{T}, \frac{\rho}{T}, \frac{\rho}{T}, \frac{\rho R}{(\gamma-1) T^{2}}\right)
$$

where

$$
q_{v}=\left(\begin{array}{rrrrr}
1 & 0 & 0 & 0 & 0 \\
v_{1} & \rho & 0 & 0 & 0 \\
v_{2} & 0 & \rho & 0 & 0 \\
v_{3} & 0 & 0 & \rho & 0 \\
-R T+\frac{1}{2} v_{1}^{2}+\frac{1}{2} v_{2}^{2}+\frac{1}{2} v_{3}^{2}+h & \rho v_{1} & \rho v_{2} & \rho v_{3} & \frac{R \rho}{\gamma-1}
\end{array}\right) .
$$

A sufficient condition to ensure that $\zeta^{T} w_{q} \zeta>0$ is to ensure that all diagonal terms above are positive. This is satisfied by $\rho, T>0$. The inverse, $S_{q q}^{-1}$ is also calculated using an expansion, $q_{w}=q_{v} v_{w}$, where

$$
v_{w}=\left(\begin{array}{rrrrrr}
\frac{\rho}{R} & \frac{\rho u_{1}}{R} & \frac{\rho u_{2}}{R} & \frac{\rho u_{3}}{R} & -\frac{2 R T \rho-\rho u_{1}^{2}-\rho u_{2}^{2}-\rho u_{3}^{2}-2 h \rho}{2 R} \\
0 & T & 0 & 0 & T u_{1} \\
0 & 0 & T & 0 & T u_{2} \\
0 & 0 & 0 & T & T u_{3} \\
0 & 0 & 0 & 0 & T^{2}
\end{array}\right) .
$$

\section{B.2 Viscous Stability}

To develop the viscous coefficient matrices used to define the viscous fluxes, the definition of the Cartesian viscous fluxes based on the primitive variable gradients is examined,

$$
f^{(v) i}=\left(0, \tau_{i 1}, \tau_{i 2}, \tau_{i 3}, \tau_{j i} v_{j}-q_{i}\right)^{T}, \quad i=1,2,3 .
$$


This can be expressed as $f^{(v) i}=c_{i j}^{\prime} v_{x_{j}}$, where

$$
\begin{aligned}
& c_{11}^{\prime}=\left(\begin{array}{ccccc}
0 & 0 & 0 & 0 & 0 \\
0 & \frac{4}{3} \mu & 0 & 0 & 0 \\
0 & 0 & \mu & 0 & 0 \\
0 & 0 & 0 & \mu & 0 \\
0 & \frac{4}{3} \mu v_{1} & \mu v_{2} & \mu v_{3} & \kappa
\end{array}\right), \quad c_{12}^{\prime}=\left(\begin{array}{ccccc}
0 & 0 & 0 & 0 & 0 \\
0 & 0 & -\frac{2}{3} \mu & 0 & 0 \\
0 & \mu & 0 & 0 & 0 \\
0 & 0 & 0 & 0 & 0 \\
0 & \mu v_{2} & -\frac{2}{3} \mu v_{1} & 0 & 0
\end{array}\right), \\
& c_{13}^{\prime}=\left(\begin{array}{ccccc}
0 & 0 & 0 & 0 & 0 \\
0 & 0 & 0 & -\frac{2}{3} \mu & 0 \\
0 & 0 & 0 & 0 & 0 \\
0 & \mu & 0 & 0 & 0 \\
0 & \mu v_{3} & 0 & -\frac{2}{3} \mu v_{1} & 0
\end{array}\right), \quad c_{21}^{\prime}=\left(\begin{array}{ccccc}
0 & 0 & 0 & 0 & 0 \\
0 & 0 & \mu & 0 & 0 \\
0 & -\frac{2}{3} \mu & 0 & 0 & 0 \\
0 & 0 & 0 & 0 & 0 \\
0 & -\frac{2}{3} \mu v_{2} & \mu v_{1} & 0 & 0
\end{array}\right), \\
& c_{22}^{\prime}=\left(\begin{array}{ccccc}
0 & 0 & 0 & 0 & 0 \\
0 & \mu & 0 & 0 & 0 \\
0 & 0 & \frac{4}{3} \mu & 0 & 0 \\
0 & 0 & 0 & \mu & 0 \\
0 & \mu v_{1} & \frac{4}{3} \mu v_{2} & \mu v_{3} & \kappa
\end{array}\right), \quad c_{23}^{\prime}=\left(\begin{array}{ccccc}
0 & 0 & 0 & 0 & 0 \\
0 & 0 & 0 & 0 & 0 \\
0 & 0 & 0 & -\frac{2}{3} \mu & 0 \\
0 & 0 & \mu & 0 & 0 \\
0 & 0 & \mu v_{3} & -\frac{2}{3} \mu v_{2} & 0
\end{array}\right), \\
& c_{31}^{\prime}=\left(\begin{array}{ccccc}
0 & 0 & 0 & 0 & 0 \\
0 & 0 & 0 & \mu & 0 \\
0 & 0 & 0 & 0 & 0 \\
0 & -\frac{2}{3} \mu & 0 & 0 & 0 \\
0 & -\frac{2}{3} \mu v_{3} & 0 & \mu v_{1} & 0
\end{array}\right), \quad c_{32}^{\prime}=\left(\begin{array}{ccccc}
0 & 0 & 0 & 0 & 0 \\
0 & 0 & 0 & 0 & 0 \\
0 & 0 & 0 & \mu & 0 \\
0 & 0 & -\frac{2}{3} \mu & 0 & 0 \\
0 & 0 & -\frac{2}{3} \mu v_{3} & \mu v_{2} & 0
\end{array}\right), \\
& c_{33}^{\prime}=\left(\begin{array}{ccccc}
0 & 0 & 0 & 0 & 0 \\
0 & \mu & 0 & 0 & 0 \\
0 & 0 & \mu & 0 & 0 \\
0 & 0 & 0 & \frac{4}{3} \mu & 0 \\
0 & \mu v_{1} & \mu v_{2} & \frac{4}{3} \mu v_{3} & \kappa
\end{array}\right) .
\end{aligned}
$$

The symmetrized coefficient matrices are found using

$$
\hat{c}_{i j}=c_{i j} q_{w}=c_{i j}^{\prime} v_{w}
$$


and thus take the form,

$$
\begin{aligned}
& \hat{c}_{11}=\left(\begin{array}{rrrrr}
0 & 0 & 0 & 0 & 0 \\
0 & \frac{4}{3} T \mu & 0 & 0 & \frac{4}{3} T \mu v_{1} \\
0 & 0 & T \mu & 0 & T \mu v_{2} \\
0 & 0 & 0 & T \mu & T \mu v_{3} \\
0 & \frac{4}{3} T \mu v_{1} & T \mu v_{2} & T \mu v_{3} & T^{2} \kappa+\frac{1}{3}\left(4 \mu v_{1}^{2}+3 \mu v_{2}^{2}+3 \mu v_{3}^{2}\right) T
\end{array}\right), \\
& \hat{c}_{22}=\left(\begin{array}{rrrrr}
0 & 0 & 0 & 0 & 0 \\
0 & T \mu & 0 & 0 & T \mu v_{1} \\
0 & 0 & \frac{4}{3} T \mu & 0 & \frac{4}{3} T \mu v_{2} \\
0 & 0 & 0 & T \mu & T \mu v_{3} \\
0 & T \mu v_{1} & \frac{4}{3} T \mu v_{2} & T \mu v_{3} & T^{2} \kappa+\frac{1}{3}\left(3 \mu v_{1}^{2}+4 \mu v_{2}^{2}+3 \mu v_{3}^{2}\right) T
\end{array}\right) \text {, } \\
& \hat{c}_{33}=\left(\begin{array}{rrrrr}
0 & 0 & 0 & 0 & 0 \\
0 & T \mu & 0 & 0 & T \mu v_{1} \\
0 & 0 & T \mu & 0 & T \mu v_{2} \\
0 & 0 & 0 & \frac{4}{3} T \mu & \frac{4}{3} T \mu v_{3} \\
0 & T \mu v_{1} & T \mu v_{2} & \frac{4}{3} T \mu v_{3} & T^{2} \kappa+\frac{1}{3}\left(3 \mu v_{1}^{2}+3 \mu v_{2}^{2}+4 \mu v_{3}^{2}\right) T
\end{array}\right) \text {, } \\
& \hat{c}_{12}=\left(\begin{array}{rrrrr}
0 & 0 & 0 & 0 & 0 \\
0 & 0 & -\frac{2}{3} T \mu & 0 & -\frac{2}{3} T \mu v_{2} \\
0 & T \mu & 0 & 0 & T \mu v_{1} \\
0 & 0 & 0 & 0 & 0 \\
0 & T \mu v_{2} & -\frac{2}{3} T \mu v_{1} & 0 & \frac{1}{3} T \mu v_{1} v_{2}
\end{array}\right) \\
& \hat{c}_{13}=\left(\begin{array}{rrrrr}
0 & 0 & 0 & 0 & 0 \\
0 & 0 & 0 & -\frac{2}{3} T \mu & -\frac{2}{3} T \mu v_{3} \\
0 & 0 & 0 & 0 & 0 \\
0 & T \mu & 0 & 0 & T \mu v_{1} \\
0 & T \mu v_{3} & 0 & -\frac{2}{3} T \mu v_{1} & \frac{1}{3} T \mu v_{1} v_{3}
\end{array}\right) \\
& \hat{c}_{23}=\left(\begin{array}{rrrrr}
0 & 0 & 0 & 0 & 0 \\
0 & 0 & 0 & 0 & 0 \\
0 & 0 & 0 & -\frac{2}{3} T \mu & -\frac{2}{3} T \mu v_{3} \\
0 & 0 & T \mu & 0 & T \mu v_{2} \\
0 & 0 & T \mu v_{3} & -\frac{2}{3} T \mu v_{2} & \frac{1}{3} T \mu v_{2} v_{3}
\end{array}\right), \\
& \hat{c}_{21}=\hat{c}_{12}^{T}, \quad \hat{c}_{31}=\hat{c}_{13}^{T}, \quad \hat{c}_{32}=\hat{c}_{23}^{T} .
\end{aligned}
$$

For the viscous terms to be entropy dissipative,

$$
w_{x_{i}}^{T} \hat{c}_{i j} w_{x_{j}} \geq 0
$$

must be satisfied. The easiest way to ensure this is to create a larger coefficient matrix,

$$
\hat{C}=\left(\begin{array}{lll}
\hat{c}_{11} & \hat{c}_{12} & \hat{c}_{13} \\
\hat{c}_{21} & \hat{c}_{22} & \hat{c}_{23} \\
\hat{c}_{31} & \hat{c}_{32} & \hat{c}_{33}
\end{array}\right)
$$


and require

$$
\left(\zeta_{x_{1}}, \zeta_{x_{2}}, \zeta_{x_{3}}\right) \hat{C}\left(\begin{array}{l}
\zeta_{x_{1}} \\
\zeta_{x_{2}} \\
\zeta_{x_{3}}
\end{array}\right) \geq 0, \quad \forall \zeta .
$$

To show this, the Cholesky decomposition, $\hat{C}=\hat{L} \hat{D} \hat{L}^{T}$ of the $(15 \times 15)$ matrix is calculated. The diagonal terms must non-negative,

$$
\hat{D}=\left(0, \frac{4}{3} T \mu, T \mu, T \mu, T^{2} \kappa, 0,0, T \mu, T \mu, T^{2} \kappa, 0,0,0,0, T^{2} \kappa\right) .
$$

In the discrete proof, the viscous terms require the additional property that each symmetric diagonal sub-block is also positive semi-definite. This is verified by,

$$
\begin{aligned}
& \hat{c}_{11}=\hat{L}_{11} \hat{D}_{11} \hat{L}_{11}^{T}, \quad \hat{D}_{11}=\left(0, \frac{4}{3} T \mu, T \mu, T \mu, T^{2} \kappa\right), \\
& \hat{c}_{22}=\hat{L}_{22} \hat{D}_{22} \hat{L}_{22}^{T}, \quad \hat{D}_{22}=\left(0, T \mu, \frac{4}{3} T \mu, T \mu, T^{2} \kappa\right), \\
& \hat{c}_{33}=\hat{L}_{33} \hat{D}_{33} \hat{L}_{33}^{T}, \quad \hat{D}_{33}=\left(0, T \mu, T \mu, \frac{4}{3} T \mu, T^{2} \kappa\right) .
\end{aligned}
$$

From the above, it is clear that in order for the viscous conditions to be satisfied,

$$
T>0, \quad \mu(T)>0, \quad \kappa(T)>0 .
$$

Thus, recall that the contribution of the viscous terms to the semi-discrete entropy decay from 4.42 is

$$
\begin{aligned}
\mathbf{w}^{T} \mathcal{P} \sum_{i=1}^{3} \mathcal{P}_{x_{i}}^{-1} \Delta_{x_{i}} \overline{\mathbf{f}}^{(v) i}= & \sum_{i=1}^{3} \sum_{\ell=1}^{3} \mathbf{w}^{T} \mathcal{P} \mathcal{P}_{x_{i}}^{-1} \mathcal{B}_{x_{i}}\left[\hat{c}_{i \ell}\right] \mathcal{D}_{x_{\ell}} \mathbf{w} \\
& -\sum_{i=1}^{3} \sum_{\ell=1}^{3}\left(\mathcal{D}_{x_{i}} \mathbf{w}\right)^{T} \mathcal{P}\left[\hat{c}_{i \ell}\right]\left(\mathcal{D}_{x_{\ell}} \mathbf{w}\right) \\
& -\sum_{i=1}^{3} \sum_{\ell=1}^{3}\left(\mathcal{N}_{\ell x_{i}} \mathbf{w}\right)^{T} \mathcal{P} \mathcal{P}_{x_{i}}^{-1} \widetilde{\left[\hat{c}_{i i}\right]_{\ell}}\left(\mathcal{N}_{\ell x_{i}} \mathbf{w}\right)
\end{aligned}
$$

The last term is easily shown to be always negative. Above it was shown that $\zeta_{x_{i}} \hat{c}_{i i} \zeta_{x_{i}} \geq 0$ is satisfied in each direction. Thus, all that is required is

$$
\boldsymbol{\zeta}^{T} \mathcal{P} \mathcal{P}_{x_{i}}^{-1} \widetilde{\left[\hat{c}_{i i}\right]_{\ell}} \boldsymbol{\zeta} \geq 0, \quad \forall \boldsymbol{\zeta}
$$

Examining A13, it is clear that $\widetilde{\left[\hat{c}_{i i}\right]_{\ell}}$ is composed of convex combinations of symmetric, positive semi-definite matrices, so the full matrix will also be positive semidefinite. The diagonal matrices $\mathcal{P} \mathcal{P}_{x_{i}}^{-1}$ are positive definite and commute with $\widetilde{\left.\hat{c}_{i i}\right]_{\ell}}$. They will not effect the eigenvalues of the matrix. Thus, the requirement above holds and the last term will always dissipate entropy. The second-to-last term on 
the right side of the entropy decay rate above is only slightly more difficult. It is first reorganized using

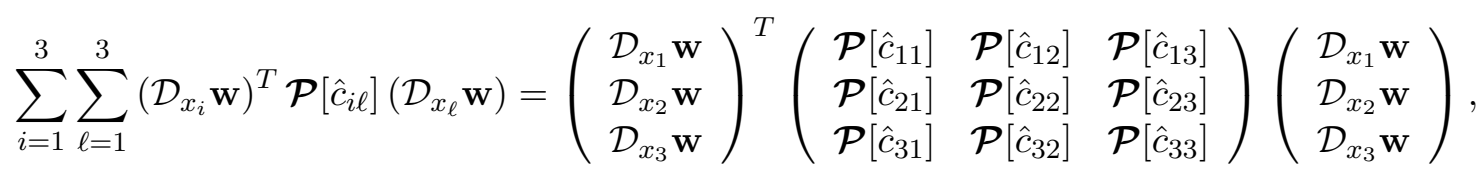

where $\mathcal{P}$ commutes with the viscous coefficient matrices, so it can be split into $\mathcal{P}=\sqrt{\mathcal{P}} \sqrt{\mathcal{P}}$ and absorbed into the gradient vectors. The gradient vectors can be reorganized such that the center term becomes

$$
\sum_{i=1}^{3} \sum_{\ell=1}^{3}\left(\mathcal{D}_{x_{i}} \mathbf{w}\right)^{T} \mathcal{P}\left[\hat{c}_{i \ell}\right]\left(\mathcal{D}_{x_{\ell}} \mathbf{w}\right)=\left(\mathbf{w}_{\mathbf{x}}\right)_{\sqrt{\mathcal{P}}}^{T}[\hat{C}]\left(\mathbf{w}_{\mathbf{x}}\right)_{\sqrt{\mathcal{P}}}
$$

where $[\hat{C}]$ is a block diagonal matrix with blocks $\hat{C}_{i}$ corresponding to the viscous coefficients at each solution point. Each of these blocks is positive semi-definite, so the full matrix is positive semi-definite. Thus,

$$
\sum_{i=1}^{3} \sum_{\ell=1}^{3}\left(\mathcal{D}_{x_{i}} \mathbf{w}\right)^{T} \mathcal{P}\left[\hat{c}_{i \ell}\right]\left(\mathcal{D}_{x_{\ell}} \mathbf{w}\right) \geq 0
$$

completing the entropy stability proof for the Navier-Stokes viscous terms. 


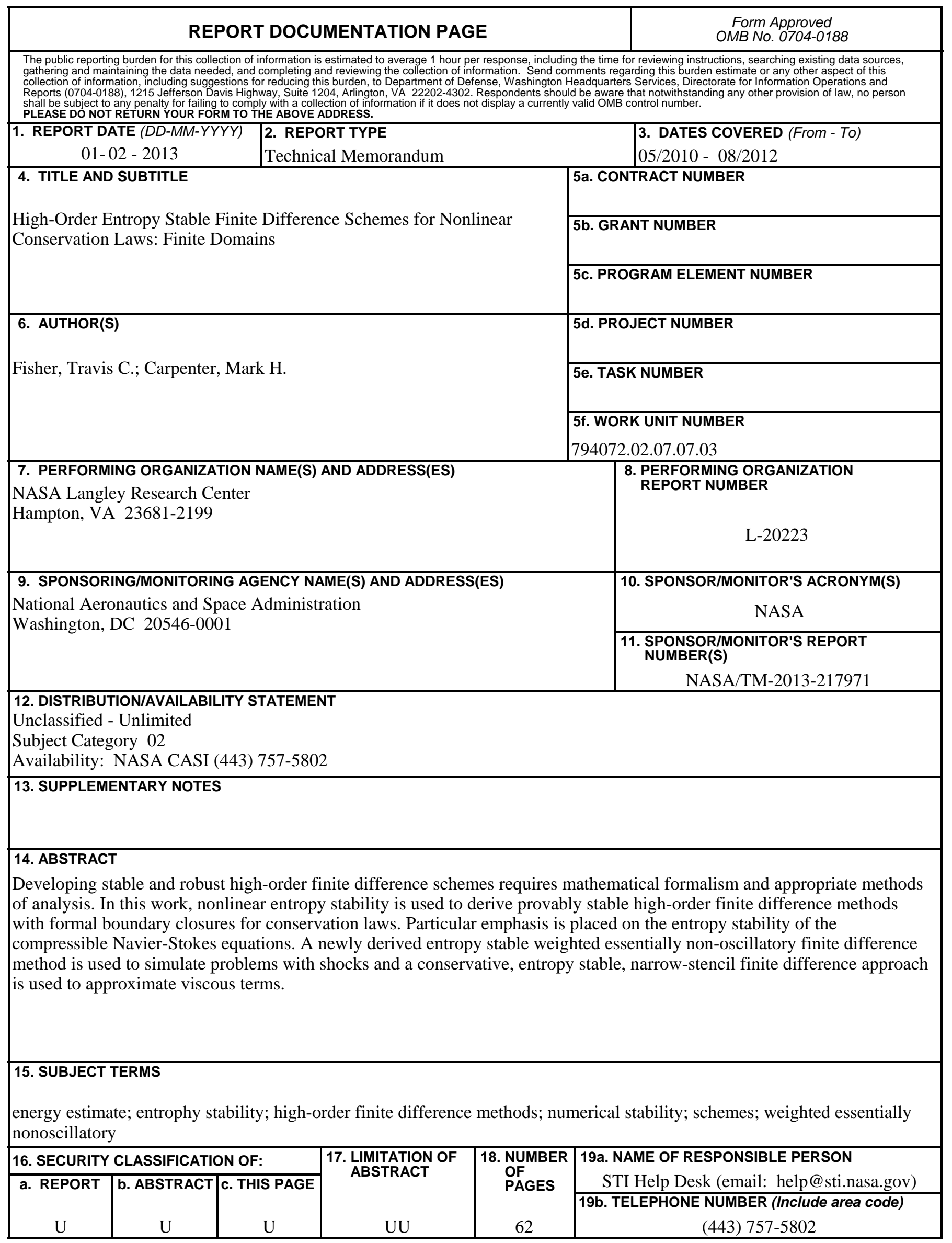

